\title{
Microscopic modeling of energy relaxation and decoherence in quantum optoelectronic devices at the nanoscale
}

\author{
D. Taj ${ }^{\mathrm{a}}$, R.C. Iotti, and F. Rossi \\ Physics Dept., Politecnico di Torino, C.so Duca degli Abruzzi 24, 10129, Torino, Italy
}

\begin{abstract}
We shall review and discuss the key problem of providing a microscopic modeling of state-ofthe-art electronic quantum devices. In particular we shall focus on the description of energy-relaxation and decoherence phenomena, explicitly showing the intrinsic limitations of some of the existing treatments (the conventional Markovian approach) via analytical results, and proposing an alternative formulation of the problem in terms of a generalized Fermi's Golden Rule.
\end{abstract}

PACS. 05.30.-d Quantum statistical mechanics - 03.65.Yz Decoherence; open systems; quantum statistical methods $-72.10 . \mathrm{Bg}$ General formulation of transport theory

\section{Introduction}

Present-day technology pushes electronic-device spaceand/or time-scales toward limits where the traditional semiclassical or Boltzmann theory [1] can no longer be applied, and more rigorous quantum-kinetic approaches are imperative [2]. However, in spite of the quantummechanical nature of electron and photon dynamics in the core region of typical solid-state nanodevices - e.g., superlattices [3] and quantum-dot structures [4] - the overall behavior of such quantum systems is often governed by a complex interplay between phase coherence and energy relaxation/dephasing [5], the latter being also due to the presence of spatial boundaries [6]. Therefore, a proper treatment of such novel nanoscale devices requires a theoretical modeling able to properly account for both coherent and incoherent - i.e., phase-breaking - processes on the same footing.

The wide family of so-called solid-state quantum devices can be schematically divided into two main classes: (i) a first one which comprises low-dimensional nanostructures whose electro-optical response may be safely treated within the semiclassical picture [7] (e.g., quantum-cascade lasers [8]) and (ii) a second one grouping solid-state devices characterized by a genuine quantum-mechanical behavior of their electronic subsystem (e.g., solid-state quantum logic gates [9]) whose quantum evolution is only weakly disturbed by decoherence processes.

For purely atomic and/or photonic quantum logic gates, decoherence phenomena are successfully described via adiabatic-decoupling procedures [10] in terms of

a Present address: Physics Dept., University of Fribourg, Chemin du Musée 3, 1700 Fribourg, Switzerland;

e-mail: david.taj@unifr.ch extremely simplified models via phenomenological parameters; within such effective treatments, the main goal/requirement is to identify a suitable form of the Liouville superoperator, able to ensure/maintain the positive-definite character of the corresponding densitymatrix operator [11]. This is usually accomplished by identifying proper Lindblad-like decoherence superoperators $[11,12]$, expressed in terms of a few crucial systemenvironment coupling parameters [13].

In contrast, solid-state devices are often characterized by a complex many-electron quantum evolution, resulting in a non-trivial interplay between coherent dynamics and energy-relaxation/decoherence processes; it follows that for a quantitative description of such coherence/dissipation coupling the latter need to be treated via fully microscopic models.

To this aim, motivated by the power and flexibility of the semiclassical kinetic theory [1] in describing a large variety of interaction mechanisms, a quantum generalization of the standard Boltzmann collision operator has been proposed [5]; the latter, obtained via the conventional Markov limit, describes the evolution of the reduced density matrix in terms of in- and out-scattering superoperators. However, contrary to the semiclassical case, such collision superoperator does not preserve the positive-definite character of the density-matrix operator.

This serious limitation was originally pointed out by Spohn and co-workers [14] three decades ago; in particular, they pointed out that the choice of the adiabatic decoupling strategy is definitely not unique, and a generic procedure does not guarantee positivity of the generated evolution. However, many groups in the physical community still keep using what is known as the Conventional Markovian (CM) approach, as it is a common belief that the associated lack of positivity actually appears only in 
extreme cases, which are non-physical or very difficult to realize experimentally (highly correlated initial states, extremely low temperatures, etc.).

With respect to this we shall employ the CM approach for a two-level quantum dot interacting with a thermal bath, and we shall show through analytical results that even at very high temperatures, such as ambient temperature or more, an arbitrarily small perturbation of the (steady) quantum dot thermal state can well trigger diverging polarizations, leading to the most drastically illdefined density matrix at large times: one with eigenvalues $1 / 2 \pm \infty$ ! This result is physically so unacceptable as to call for a new adiabatic procedure, able to guarantee positivity.

Inspired by the pioneering papers by Davies and coworkers [15], which however was severely limited to subsystem with discrete spectrum (i.e. no internal structure), the second important aim of this paper is to present an alternative and more general adiabatic procedure which (i) in the discrete spectral case reduces to Davies' model; (ii) for diagonal states gives the well known Fermi's golden rule $[16,17]$; and (iii) describes a genuine Lindblad evolution, irrespective of the subsystem's spectral properties, thus providing a reliable/robust treatment of energy-dissipation and dephasing processes in semiconductor quantum devices. We stress that, contrary to standard master-equation formulations [15,18], neither the proposed adiabatic-decoupling approach, nor the resulting Lindblad structure, depend on the particular way to choose the subsystem, be it by reduction or by other projection procedures.

It is important at this point to underline that the validity regime of our results will be exactly the same as that of the CM approach [19-21], which is currently used. Of course, there are situations in nowadays nanostructure and nanodevices where our approach is not applicable (and thus neither the CM approach), like systems which so strongly interact with the environment, that they suffer from some environment backreaction, in turn taking the system to a non-markovian regime. This is the case for example of two quantum dots which are spatially so close as to feel each other through the phonons of the bath. Another example is that of pure dephasing in quantum dot structures [22], which is known not to have a markovian limit (we shall see it explicitly in our example for a two level system). However, our approach can be applied to the entire family of markovian systems, provided that the external degrees of freedom have correlation functions which vanish, in time, sufficiently fast. This of course forces the external degrees of freedom to be at least infinite in number, and indeed to form a continuum of modes. If this was not the case, oscillations between system and environment would be observed, leaving no hope for a dissipative and irreversible evolution, even in the weak-coupling regime. How fast the bath correlation functions should decay is an important problem, technically very hard, which is outlined in general [21] and was solved in [15] in case of a free fermionic bath (not necessarily at thermal equilibrium), and a coupling to the system quadratic in the bath fields.
This latter fact shows that, in case the system is chosen by a partial trace reduction, the bath need not be at thermal equilibrium for our approach to apply: all we need, exactly as with the CM approach, is that the bath is not backreacting to the system at long times (a markovian, memoryfree hypothesis), where "long" compares to the coupling Hamiltonian (a weak-coupling hypothesis). So our model could apply equally well to, say, a bath made of coherent bosons (for example a continuous wave laser), provided that the memory free and weak-coupling assumptions are satisfied (see Thms. 7.1, 7.2 and 9.1 in [21] for a precise formulation).

The paper is organized as follows. In Section 1 we shall review the fundamentals of the density-matrix formalism applied to the analysis of solid-state quantum devices; Section 2 will provide a fully-operatorial derivation of the conventional adiabatic or Markov approximation; Section 3 will address the general derivation of effective scattering superoperators describing the electronic subsystem interacting with various quasiparticles, e.g., phonons, photons, plasmons, etc. In Section 4 we analyze the CM approach as applied to the case of a quantum dot interacting with a thermal bosonic bath, and show the intrinsic and physically non-negligible problems of the former, way down to a two-level case. In Section 5 we shall propose an alternative formulation of the Markov limit, leading to the derivation of a Lindblad-like scattering superoperator in terms of a generalized or quantum Fermi's golden rule, which is then specialized to the partial trace with a thermal bosonic bath in Section 6. In Section 7 we shall discuss the possible application of our formulation to non-conventional projection schemes, like, e.g., the treatment of quantum devices with open boundaries. Finally, in Section 8 we shall summarize and draw some conclusions.

\section{Fundamentals of the density-matrix theory applied to solid-state nanodevices}

The idealized behavior of a so-called "quantum device" [7] is usually described via the elementary physical picture of the square-well potential and/or in terms of a simple quantum-mechanical $n$-level system. For a quantitative investigation of state-of-the-art quantum optoelectronic devices, however, two features strongly influence and modify such simplified scenario: (i) the intrinsic many-body nature of the carrier system under investigation; and (ii) the potential coupling of the electronic subsystem of interest with a variety of interaction mechanisms, including the presence of spatial boundaries [6]. These systems are characterized by a strong interplay between coherent dynamics and energy-relaxation/dephasing processes; it follows that for a quantitative description of such non-trivial coherence/dissipation coupling the latter need to be treated via fully microscopic models.

In this paper we shall provide a comprehensive microscopic theory of charge quantum transport in semiconductor nanostructures based on the well-known density-matrix approach. It is worth mentioning that an 
alternative approach, equivalent to the density-matrix formalism employed in our work, is given by the nonequilibrium Green's function technique; the latter can be regarded as an extension of the well-known equilibrium or zero-temperature Green's function theory to nonequilibrium regimes, introduced in the 1960 s by Kadanoff and Baym [23] and Keldysh [24]. An introduction to the theory of nonequilibrium Green's functions with applications to many problems in transport and optics of semiconductors can be found in the book by Haug and Jauho [25]. By employing - and further developing and extending such nonequilibrium Green's function formalism, a number of groups have proposed efficient quantum-transport treatments for the study of various semiconductor nanostructures as well as of modern micro/optoelectronic devices $[26]$.

Within the general density-matrix formalism two different strategies are commonly employed: (i) the quantumkinetic treatment [5]; and (ii) the description based on the Liouville-von Neumann equation [27].

The primary goal of a quantum-kinetic theory is to evaluate the temporal evolution of a reduced set of singleor few-particle quantities directly related to the electrooptical phenomenon under investigation, the so-called kinetic variables of the system. However, due to the manybody nature of the problem, an exact solution in general is not possible; it follows that for a detailed understanding realistic semiconductor models have to be considered, which then can only be treated approximately. Within the kinetic-theory approach one starts directly with the equations of motion for the single-particle density matrix. Due to the many-body nature of the problem, the resulting set of equations of motion is not closed; instead, it constitutes the starting point of an infinite hierarchy of higher-order density matrices. Besides differences related to the quantum statistics of the quasiparticles involved, this is equivalent to the BBGKY hierarchy in classical gas dynamics [28]. The central approximation in this formalism is the truncation of the hierarchy. This can be based on different physical pictures. A common approach is to use the argument that correlations involving an increasing number of particles will become less and less important [5]. An alternative quantum-kinetic scheme - based on an expansion in powers of the exciting electromagnetic field - has been introduced by Axt and Stahl, the so-called "dynamics controlled truncation" (DCT) [29].

Within the treatment based on the Liouville-von Neumann equation, the starting point is the equation of motion for the global density-matrix operator, describing many electron plus various quasiparticle excitations. The physical quantities of interest for the electronic subsystem are then typically derived via a suitable "reduction procedure", aimed at tracing out non-relevant degrees of freedom. Contrary to the kinetic theory, this approach has allowed for a fully quantum-mechanical treatment of highfield transport in semiconductors [30], thus overcoming some of the basic limitations of conventional kinetic treatments, e.g., the completed-collision limit and the Markov approximation.
As anticipated, primary goal of the present paper is to discuss in very general terms the physical properties and validity limits of the so-called "adiabatic" or Markov approximation. Within the traditional semiclassical or Boltzmann theory, this approximation is typically introduced together with the so-called diagonal approximation, i.e., the neglect of non-diagonal density-matrix elements. However, as described in reference [5], the Markov limit can be also performed within a fully non-diagonal densitymatrix treatment of the problem; this leads to the introduction of generalized in- and out-scattering superoperators, whose general properties and physical interpretation are not straightforward. In particular, it is imperative to understand if - and under which conditions - the adiabatic or Markov approximation preserves the positive-definite character of our reduced density matrix; indeed, this distinguished property is generally lost within the quantumkinetic approaches previously mentioned $[5,31]$.

\section{Operatorial derivation of the conventional Markov approximation}

In order to discuss the main features and intrinsic limitations of the conventional adiabatic or Markov limit, let us recall its general derivation following the fully operatorial approach proposed in reference [32]. Given a generic physical quantity $\hat{A}$ - described by the operator $A$ - its quantum plus statistical average value is given by

$$
\langle\hat{A}\rangle=\operatorname{tr}\{A \rho\},
$$

where $\rho$ is the so-called density-matrix operator. Its time evolution is dictated by the total (system plus environment) Hamiltonian. Within the usual weak-coupling scheme, the latter can be regarded as the sum of a noninteracting (system plus environment) contribution plus a system-environment coupling term, weighted by a small dimensionless coupling constant $\lambda$. Written in units of $\hbar$, this reads:

$$
H=H_{\circ}+\lambda H^{\prime} .
$$

The corresponding equation of motion for the densitymatrix operator - also known as Liouville-von Neumann equation - in the interaction picture is given by:

$$
\frac{d \rho^{i}}{d t}(t)=-i \lambda\left[H^{i}(t), \rho^{i}(t)\right],
$$

where $H^{i}(t)$ denotes the interaction Hamiltonian $H^{\prime}$ written at current time $t$.

We stress that - according to the standard procedure at this point one should identify a subsystem of interest by, for example, the use of a suitable projection on the space of density matrices: although this step is crucial to study the weak-coupling limit, it turns out, as we shall see, that it needs to be considered explicitly only later on, when talking about positivity ${ }^{1}$. Hence for ease of exposition we

\footnotetext{
1 For the moment, we shall limit ourselves to use the projection $P_{0}$ (defined in the Heisenberg picture), to say that no first-order terms are considered in this paper, i.e., we shall assume that $P_{0}\left(\left[H^{\prime}(t), P_{0} A\right]\right)=0$.
} 
shall first focus on all the projection-independent features of our theoretical framework.

The key idea beyond any perturbation approach is that the effect of the interaction Hamiltonian $H^{\prime}$ is "small" compared to the free evolution dictated by the noninteracting Hamiltonian $H_{\circ}$, that is, the coupling constant $\lambda$ is small. Following this spirit, by formally integrating equation (3) from $t_{\circ}$ to the current time $t$, and inserting such formal solution for $\rho^{i}(t)$ on the right-hand side of equation (3), we obtain an integro-differential equation of the form:

$$
\begin{aligned}
\frac{d}{d t} \rho^{i}(t)= & -i \lambda\left[H^{i}(t), \rho^{i}\left(t_{\circ}\right)\right] \\
& -\lambda^{2} \int_{t_{\circ}}^{t} d t^{\prime}\left[H^{i}(t),\left[H^{i}\left(t^{\prime}\right), \rho^{i}\left(t^{\prime}\right)\right]\right] .
\end{aligned}
$$

We stress that so far no approximation has been introduced: equations (3) and (4) are fully equivalent, we have just isolated the first-order contribution from the full time evolution in equation (3).

Let us now focus on the time integral in equation (4). Here, the two quantities to be integrated over $t^{\prime}$ are the interaction Hamiltonian $H^{i}$ and the density-matrix operator $\rho^{i}$. In the spirit of the perturbation approach previously recalled, the time variation of $\rho^{i}$ can be considered adiabatically slow compared to that of the Hamiltonian $H^{i}$ written in the interaction picture, i.e.,

$$
H^{i}\left(t^{\prime}\right)=U_{\circ}^{\dagger}\left(t^{\prime}\right) H^{\prime} U_{\circ}\left(t^{\prime}\right)
$$

indeed, the latter exhibits rapid oscillations due to the noninteracting evolution operator

$$
U_{\circ}(t)=e^{-i H_{\circ} t} .
$$

As a result, the density-matrix operator $\rho^{i}$ can be taken out of the time integral and evaluated at the current time $t$.

Following such prescription, the second-order contribution to the system dynamics written in the Schrödinger picture for the case of a time-independent interaction Hamiltonian $H^{\prime}$ comes out to be:

$$
\frac{d \rho}{d t}=-\lambda^{2}\left[H^{\prime},[\mathcal{K}, \rho]\right]
$$

with

$$
\mathcal{K}=\int_{0}^{t-t_{\circ}} d t^{\prime} \mathcal{H}^{i}\left(t^{\prime}\right)=\int_{0}^{t-t_{\circ}} d t^{\prime} U_{\circ}^{\dagger}\left(t^{\prime}\right) \mathcal{H}^{\prime} U_{\circ}\left(t^{\prime}\right) .
$$

The effective equation (7) has still the double-commutator structure of equation (4) but it is now local in time. The Markov limit recalled so far leads to significant modifications to the system dynamics: while the exact quantummechanical evolution in equation (3) corresponds to a fully reversible and isoentropic unitary transformation, the instantaneous double-commutator structure in equation (7) describes, in general, a non-reversible (i.e., non unitary) dynamics characterized by energy dissipation and dephasing.
First of all, let us now show that the master equation (7) constitutes a generalization of the classical Fermi's Golden Rule, as it boils down to the latter in the diagonal case. By denoting with $\{|\lambda\rangle\}$, and neglecting energy renormalization contributions [32], the eigenstates of the noninteracting Hamiltonian $\hat{H}_{\circ}$, the effective equation (7) written in this basis is of the form:

$$
\frac{d \rho_{\lambda_{1} \lambda_{2}}}{d t}=\frac{1}{2} \sum_{\lambda_{1}^{\prime} \lambda_{2}^{\prime}}\left[\mathcal{P}_{\lambda_{1} \lambda_{2}, \lambda_{1}^{\prime} \lambda_{2}^{\prime}} \rho_{\lambda_{1}^{\prime} \lambda_{2}^{\prime}}-\mathcal{P}_{\lambda_{1} \lambda_{2}^{\prime}, \lambda_{1}^{\prime} \lambda_{1}^{\prime}} \rho_{\lambda_{2}^{\prime} \lambda_{2}}\right]+\text { H.c. }
$$

with generalized scattering rates given by:

$$
\mathcal{P}_{\lambda_{1} \lambda_{2}, \lambda_{1}^{\prime} \lambda_{2}^{\prime}}=\frac{2 \pi}{\hbar} H_{\lambda_{1} \lambda_{1}^{\prime}}^{\prime} H_{\lambda_{2} \lambda_{2}^{\prime}}^{\prime *} \delta\left(\epsilon_{\lambda_{2}}-\epsilon_{\lambda_{2}^{\prime}}\right)
$$

$\epsilon_{\lambda}$ denoting the energy corresponding to the noninteracting state $|\lambda\rangle$. As discussed extensively in [32], such generalized scattering rates are obtained within the completed-collision limit, i.e., $t_{\circ} \rightarrow-\infty$, and neglecting energy-renormalization contributions.

The well-known semiclassical or Boltzmann theory [1] can be easily derived from the quantum-transport formulation presented so far, by introducing the so-called diagonal or semiclassical approximation. The latter corresponds to neglecting all non-diagonal density-matrix elements (and therefore any quantum-mechanical phase coherence between the generic states $\lambda_{1}$ and $\lambda_{2}$ ), i.e., $\rho_{\lambda_{1} \lambda_{2}}=f_{\lambda_{1}} \delta_{\lambda_{1} \lambda_{2}}$, where the diagonal elements $f_{\lambda}$ describe the semiclassical distribution function over our noninteracting basis states. Within such approximation scheme, the quantum-transport equation (122) reduces to the wellknown Boltzmann equation:

$$
\frac{d f_{\lambda}}{d t}=\sum_{\lambda^{\prime}}\left(P_{\lambda \lambda^{\prime}} f_{\lambda^{\prime}}-P_{\lambda^{\prime} \lambda} f_{\lambda}\right)
$$

where

$$
P_{\lambda \lambda^{\prime}}=\mathcal{P}_{\lambda \lambda, \lambda^{\prime} \lambda^{\prime}}=\frac{2 \pi}{\hbar}\left|H_{\lambda \lambda^{\prime}}^{\prime}\right|^{2} \delta\left(\epsilon_{\lambda}-\epsilon_{\lambda^{\prime}}\right)
$$

are the conventional semiclassical scattering rates given by the well-known Fermi's golden rule [16].

At this point it is crucial to stress that, contrary to the non-diagonal density-matrix description previously introduced, the Markov limit combined with the semiclassical or diagonal approximation ensures that at any time $t$ our semiclassical distribution function $f_{\lambda}$ is always positive-definite. This explains the "robustness" of the Boltzmann transport equation (125), and its extensive application in solid-state-device modeling as well as in many other areas, where quantum effects play a very minor role. In contrast, in order to investigate genuine quantummechanical phenomena, the conventional Markov superoperator in (7) cannot be employed, since it does not preserve the positive-definite character of the density ma$\operatorname{trix} \rho_{\lambda_{1} \lambda_{2}}$.

Since any effective Liouville superoperator should describe correctly the time evolution of $\rho$ and since the latter, by definition, needs to be trace-invariant and positivedefinite at any time, it is imperative to determine if the 
Markov superoperator in equation (7) fulfills this two basic requirements. As far as the first issue is concerned, in view of its commutator structure, it is easy to show that this effective superoperator is indeed trace-preserving.

In contrast, it is not at all obvious a priori that for any initial condition the density-matrix operator will be positive-definite at any time. Indeed, as originally shown in reference [12], this fulfillment is accomplished by employing a local superoperator of the form:

$$
\frac{d}{d t} \rho=-\sum_{i}\left(\frac{1}{2}\left\{L_{i}^{\dagger} L_{i}, \rho\right\}+L_{i} \rho L_{i}^{\dagger}\right),
$$

where $\left\{L_{i}\right\}$ is a generic set of operators. The latter, known as Lindblad superoperator, for the particular case of Hermitian operators (i.e. $L_{i}=L_{i}^{\dagger}$ ) reduces to:

$$
\frac{d}{d t} \rho=-\frac{1}{2} \sum_{i}\left[L_{i},\left[L_{i}, \rho\right]\right] .
$$

We stress that the Markov superoperator in equation (7) exhibits the same double-commutator structure in equation (14). However, due to its strongly asymmetric form, the latter is not cast in an explicit Lindblad form. This is by far the most severe limitation of the conventional Markov approximation: we shall prove in the following that the conventional Markov (CM) superoperator does not preserve a positive evolution, in a physically nonnegligible way.

\section{Partial trace projection on the electronic subsystem}

As anticipated, the whole theoretical scheme described so far becomes meaningful and applicable only when a welldefined subsystem of interest is identified (together with a corresponding infinite-dimensional environment). To this end, let us now specialize to the case of a semiconductor quantum device, for which the unperturbed Hamiltonian $H_{\circ}$ in equation (2) can be written as

$$
H_{\circ}=H_{e}+H_{b} \text {. }
$$

This is the sum of the electronic $(e)$ Hamiltonian and of the free-bath (b) Hamiltonian

$$
H_{b}=\int d \mathbf{q} \omega_{b}(\mathbf{q}) b_{\mathbf{q}}^{\dagger} b_{\mathbf{q}}
$$

where the Bosonic operators $b_{\mathbf{q}}^{\dagger}\left(b_{\mathbf{q}}\right)$ denote creation (destruction) of a generic quasiparticle excitation with wavevector $\mathbf{q}$ and energy $\epsilon_{\mathbf{q}}=\hbar \omega_{b}(\mathbf{q})$, i.e., phonons, photons, plasmons, etc.

In this case, the noninteracting (carrier-plusquasiparticle) basis states

$$
|\lambda\rangle=|\alpha\rangle \otimes\left|\left\{n_{\mathbf{q}}\right\}\right\rangle
$$

are given by the tensor product of electronic states $|\alpha\rangle$ and quasiparticle states $\left|\left\{n_{\mathbf{q}}\right\}\right\rangle$ corresponding to the occupation density $\left\{n_{\mathbf{q}}\right\}$; the noninteracting energy spectrum

$$
\omega_{\lambda}=\omega_{\alpha}+\int d \mathbf{q} \omega_{b}(\mathbf{q}) n_{\mathbf{q}}
$$

is then the sum of the electronic and total quasiparticle energies.

For all relevant carrier-quasiparticle interaction mechanisms in semiconductor nanostructures - e.g., carrierphonon, carrier-photon, carrier-plasmon, etc. - the perturbation Hamiltonian $H^{\prime}$ in equation (2) can be written as:

$$
H^{\prime}=\int d \mathbf{q}\left(H_{\mathbf{q}} b_{\mathbf{q}}+H_{\mathbf{q}}^{\dagger} b_{\mathbf{q}}^{\dagger}\right)=H^{a b}+H^{e m} .
$$

Here $H_{\mathbf{q}}=H_{-\mathbf{q}}^{\dagger}$ are electronic operators (parameterized by the quasiparticle wavevector $\mathbf{q}$ ) acting on the $\alpha$ subsystem only. The two terms in equation (19) - corresponding to quasiparticle destruction and creation - describe electronic absorption and emission processes.

As anticipated, the average value of any given physical quantity $A$ can be easily expressed in terms of the density-matrix operator $\rho$ according to equation (1). In the study of electronic quantum phenomena in semiconductor nanostructures, most of the physical quantities of interest depend on the electronic-subsystem coordinates $\alpha$ only (carrier energy loss, electronic coherence length, carrier-carrier correlation functions, etc.), i.e.,

$$
A_{\lambda \lambda^{\prime}}=A_{\alpha,\left\{n_{\mathbf{q}}\right\} ; \alpha^{\prime},\left\{n_{\mathbf{q}}^{\prime}\right\}}=A_{\alpha \alpha^{\prime}} \delta_{\left\{n_{\mathbf{q}}\right\}\left\{n_{\mathbf{q}}^{\prime}\right\}} \cdot
$$

In this case it is convenient to write equation (1) as

$$
A=\operatorname{tr}\{A \rho\}=\sum_{\lambda \lambda^{\prime}} A_{\lambda \lambda^{\prime}} \rho_{\lambda^{\prime} \lambda}=\sum_{\alpha, \alpha^{\prime}} A_{\alpha \alpha^{\prime}} \rho_{\alpha^{\prime} \alpha}^{e}
$$

where

$$
\rho_{\alpha \alpha^{\prime}}^{e}=\sum_{\left\{n_{\mathbf{q}}\right\}} \rho_{\alpha,\left\{n_{\mathbf{q}}\right\} ; \alpha^{\prime},\left\{n_{\mathbf{q}}\right\}}
$$

is the so-called reduced or electronic density matrix. Equation (22) can be also written in an operatorial form as:

$$
\rho^{e}=\operatorname{tr}\{\rho\}_{b},
$$

which shows that the electronic density-matrix operator $\rho^{e}$ is obtained by performing a trace operation over the quasiparticle variables $\left\{n_{\mathbf{q}}\right\}$. Since $\rho^{e}$ is the only quantity entering the evaluation of the average value in equation (21), it is desirable to derive a corresponding equation of motion for the reduced density-matrix operator. However, such trace over the quasiparticle coordinates does not commute with the scattering superoperator one wants to project, a fact which does not allow to obtain a closed equation of motion for the reduced density-matrix operator $\rho^{e}$. In order to overcome this problem, the typical assumption is to consider the quasiparticle subsystem as characterized by a huge number of degrees of freedom (compared to the 
subsystem $\alpha$ ). In other words this amounts to say that the quasiparticle subsystem has an infinitely high heat capacity, i.e., it behaves as a thermal bath; this allows to consider this subsystem always in thermal equilibrium, i.e., not significantly perturbed by the electronic subsystem. Within such approximation scheme, the global ( $e$ plus $b$ ) density-matrix operator $\rho$ can be written as the product of the equilibrium density-matrix operator for the quasiparticle subsystem $\rho^{b}$ and the reduced density-matrix operator $\rho^{e}$ :

$$
\rho=\rho^{e} \otimes \rho^{b}, \quad \rho^{b}=\frac{e^{-\frac{\hbar H_{b}}{k_{\mathrm{B}} T}}}{\operatorname{tr}\left\{e^{-\frac{\hbar H_{b}}{k_{\mathrm{B}} T}}\right\}} .
$$

This bath state can also implies, and can also be abstractly defined by, the following two-point bath correlation functions:

$$
\operatorname{tr}\left\{b_{\mathbf{q}} b_{\mathbf{q}^{\prime}} \rho^{b}\right\}=\operatorname{tr}\left\{b_{\mathbf{q}}^{\dagger} b_{\mathbf{q}^{\prime}}^{\dagger} \rho^{b}\right\}=0,
$$

and

$$
N_{\mathbf{q}}=\operatorname{tr}\left\{b_{\mathbf{q}}^{\dagger} b_{\mathbf{q}} \rho^{b}\right\},
$$

where $N_{\mathbf{q}}$ denotes the average occupation density for the quasiparticle state $\mathbf{q}$.

It is important to note that all this reduction scheme can be easily implemented by using a projection $P$, that acts on a generic global density matrix to give the partial trace through

$$
P \rho=\operatorname{tr}\{\rho\}_{b} \otimes \rho^{b}=\rho^{e} \otimes \rho^{b},
$$

which clearly establishes the relationship between global and subsystem density matrix. Moreover, this projection is superoperatorial in nature, as it acts on operators (density matrices). It allows to explicitly write a projected effective Markov superoperator $\mathbb{L}$ in the following simple way

$$
\partial_{t} P \rho=P \mathbb{L} P \rho .
$$

Recalling the explicit form of the noninteracting Hamiltonian $H_{\circ}$ in equation (15), we have:

$$
\begin{aligned}
U_{\circ}\left(t^{\prime}\right) & =e^{-i H_{\circ} t^{\prime}} \\
& =e^{-i H_{e} t^{\prime}} e^{-i H_{b} t^{\prime}} \\
& =U_{e}\left(t^{\prime}\right) U_{q p}\left(t^{\prime}\right) .
\end{aligned}
$$

Moreover, employing the bosonic commutation relations for the creation and destruction operators, we get:

$$
\begin{aligned}
b_{\mathbf{q}}^{i}(t) & =U_{b}^{\dagger}(t) b_{\mathbf{q}} U_{b}(t)=e^{-i \omega_{b}(\mathbf{q}) t} b_{\mathbf{q}} \\
b_{\mathbf{q}}^{i \dagger}\left(t^{\prime}\right) & =U_{b}^{\dagger}(t) b_{\mathbf{q}}^{\dagger} U_{b}(t)=e^{+i \omega_{b}(\mathbf{q}) t} b_{\mathbf{q}}^{\dagger} .
\end{aligned}
$$

In view of these two properties, the explicit form of the electron-quasiparticle coupling Hamiltonian in equation (19) written in the interaction picture comes out to be:

$$
H^{i}\left(t^{\prime}\right)=\int d \mathbf{q}\left(H_{\mathbf{q}}^{-}\left(t^{\prime}\right) b_{\mathbf{q}}+H_{\mathbf{q}}^{+}\left(t^{\prime}\right) b_{\mathbf{q}}^{\dagger}\right)
$$

with

$$
H_{\mathbf{q}}^{-}(t)=H_{\mathbf{q}}^{i}(t) e^{-i \omega_{b}(\mathbf{q}) t}, \quad H_{\mathbf{q}}^{+}(t)=H_{\mathbf{q}}^{i \dagger}(t) e^{+i \omega_{b}(\mathbf{q}) t} .
$$

We also note that $H_{\mathbf{q}}^{ \pm}\left(t^{\prime}\right)=H_{\mathbf{q}}^{\mp \dagger}\left(t^{\prime}\right)$, as concerns the interaction. As we can see, for each quasiparticle state/mode q we have always two contributions $( \pm)$ describing quasiparticle emission and absorption.

\section{Conventional Markov for a quantum dot}

We start with our projected CM superoperator, which has the general form

$$
\partial_{t} P \rho=-i\left[H_{0}, P \rho\right]-\lambda^{2} P\left(\left[H^{\prime},[K, P \rho]\right]\right) .
$$

We choose the usual free evolution

$$
\begin{aligned}
H_{\circ} & =H_{a} \otimes 1+1 \otimes H_{b} \\
& =\sum_{\alpha} \omega_{\alpha} c_{\alpha}^{\dagger} c_{\alpha} \otimes 1+1 \otimes \int d \mathbf{q} \omega_{b}(\mathbf{q}) b_{\mathbf{q}}^{\dagger} b_{\mathbf{q}}
\end{aligned}
$$

and interaction of the type

$$
\begin{aligned}
H^{\prime} & =\sum_{\alpha \beta} \int d \mathbf{q} g_{\alpha \beta}(\mathbf{q}) c_{\alpha}^{\dagger} c_{\beta} \otimes b_{\mathbf{q}}+\text { h.c. } \\
& =\sum_{\alpha \beta} c_{\alpha}^{\dagger} c_{\beta} \otimes \int d \mathbf{q}\left(g_{\alpha \beta}(\mathbf{q}) b_{\mathbf{q}}+g_{\beta \alpha}(\mathbf{q})^{*} b_{\mathbf{q}}^{\dagger}\right)
\end{aligned}
$$

that is, $H_{\mathbf{q}}=\sum_{\alpha \beta} g_{\alpha \beta}(\mathbf{q}) c_{\alpha}^{\dagger} c_{\beta}$, for a generic complexvalued coupling coefficient $g_{\alpha \beta}(\mathbf{q})$, given as a function of the wavevector $\mathbf{q}$.

The latter is the prototypical Hamiltonian describing generic relaxation processes in a semiconductor quantum dot via bosonic quasiparticle interaction mechanisms (i.e., thermal phonons), and thus constitutes a most relevant physical scenario.

To compute $K$ we denote $\omega_{\alpha \beta}=\omega_{\alpha}-\omega_{\beta}$, and we compute

$$
\begin{aligned}
H^{\prime}(-t) & =e^{-i \omega_{\alpha \beta} t} c_{\alpha}^{\dagger} c_{\beta} \otimes \\
& \otimes \int d \mathbf{q}\left(e^{i \omega_{b}(\mathbf{q}) t} g_{\alpha \beta}(\mathbf{q}) b_{\mathbf{q}}+e^{-i \omega_{b}(\mathbf{q}) t} g_{\beta \alpha}(\mathbf{q})^{*} b_{\mathbf{q}}^{\dagger}\right) .
\end{aligned}
$$

Then, putting $P \rho=\rho^{e} \otimes \rho^{b}$, we need to compute four terms, namely

1. $-P\left(H^{\prime} K \rho^{e} \otimes \rho^{b}\right)$;

2. $-P\left(\rho^{e} \otimes \rho^{b} K H^{\prime}\right)$;

3. $P\left(H^{\prime} \rho^{e} \otimes \rho^{b} K\right)$;

4. $P\left(K \rho^{e} \otimes \rho^{b} H^{\prime}\right)$.

To compute the four terms above, we note that both $H^{\prime}$ and $K$ contain two contributions, coming from the annihilation and the creation bosonic operator, respectively. Due to the chosen projection, and to the two-point bath correlation function properties stated above, we shall drop the products of two creation contributions, as well as two 
annihilation ones, as these will not contribute to the four terms above. Then, according to the relative order among the creation and annihilation contribution, as well as the bath density matrix $\sigma$, we shall obtain the appropriate emission and absorption parts.

We compute (1.) as follows (we omit to again tensor product with $\rho^{b}$ in the final result, which would be formally required):

$$
P\left(H^{\prime} K \rho^{e} \otimes \rho^{b}\right)=B_{\alpha_{1} \beta_{1} \alpha_{2} \beta_{2}} c_{\alpha_{1}}^{\dagger} c_{\beta_{1}} c_{\alpha_{2}}^{\dagger} c_{\beta_{2}} \rho^{e}
$$

with

$$
\begin{aligned}
B_{\alpha_{1} \beta_{1} \alpha_{2} \beta_{2}}=2 & \pi \int d \mathbf{q}\left\{g_{\alpha_{1} \beta_{1}}(\mathbf{q}) g_{\beta_{2} \alpha_{2}}(\mathbf{q})^{*}\left(N_{\omega_{b}(\mathbf{q})}+1\right) \mathcal{D}_{\alpha_{2} \beta_{2}, \mathbf{q}}^{+}\right. \\
& \left.+g_{\beta_{1} \alpha_{1}}(\mathbf{q})^{*} g_{\alpha_{2} \beta_{2}}(\mathbf{q}) N_{\omega_{b}(\mathbf{q})} \mathcal{D}_{\alpha_{2} \beta_{2}, \mathbf{q}}^{-}\right\}, \quad(38)
\end{aligned}
$$

where, just as in [32], we have defined

$$
\begin{aligned}
\mathcal{D}_{\alpha \beta, \mathbf{q}}^{ \pm} & =\frac{1}{2 \pi} \int_{0}^{\infty} d t e^{-i\left(\omega_{\alpha \beta} \pm \omega_{b}(\mathbf{q})\right) t} \\
& =\frac{1}{2} \delta\left(\omega_{\alpha \beta} \pm \omega_{b}(\mathbf{q})\right)+\frac{1}{2 \pi i} \frac{1}{\omega_{\alpha \beta} \pm \omega_{b}(\mathbf{q})}
\end{aligned}
$$

In passing, we recall the property $\mathcal{D}_{\alpha \beta, \mathbf{q}}^{ \pm, *}:=\left(\mathcal{D}_{\alpha \beta, \mathbf{q}}^{ \pm}\right)^{*}=$ $\mathcal{D}_{\beta \alpha, \mathbf{q}}^{\mp}$.

Now we compute (2.) as follows:

$$
P\left(\rho^{e} \otimes \rho^{b} K H^{\prime}\right)=\rho^{e} c_{\alpha_{1}}^{\dagger} c_{\beta_{1}} c_{\alpha_{2}}^{\dagger} c_{\beta_{2}} C_{\alpha_{1} \beta_{1} \alpha_{2} \beta_{2}}
$$

with

$$
\begin{array}{rl}
C_{\alpha_{1} \beta_{1} \alpha_{2} \beta_{2}}=2 & 2 \pi \int d \mathbf{q}\left\{g_{\alpha_{1} \beta_{1}}(\mathbf{q}) g_{\beta_{2} \alpha_{2}}(\mathbf{q})^{*}\left(N_{\omega_{b}(\mathbf{q})}+1\right) \mathcal{D}_{\alpha_{1} \beta_{1}, \mathbf{q}}^{-}\right. \\
& \left.+g_{\beta_{1} \alpha_{1}}(\mathbf{q})^{*} g_{\alpha_{2} \beta_{2}}(\mathbf{q}) N_{\omega_{b}(\mathbf{q})} \mathcal{D}_{\alpha_{1} \beta_{1}, \mathbf{q}}^{+}\right\} .
\end{array}
$$

We compute (3.) as follows (note the index exchange in the coefficient $C$ with respect to before):

$$
P\left(H^{\prime} \rho^{e} \otimes \rho^{b} K\right)=c_{\alpha_{1}}^{\dagger} c_{\beta_{1}} \rho^{e} c_{\alpha_{2}}^{\dagger} c_{\beta_{2}} C_{\alpha_{2} \beta_{2} \alpha_{1} \beta_{1}} .
$$

Finally, we compute (4.) as follows (again, index exchange for the coefficient $B$ ):

$$
P\left(K \rho^{e} \otimes \rho^{b} H^{\prime}\right)=c_{\alpha_{1}}^{\dagger} c_{\beta_{1}} \rho^{e} c_{\alpha_{2}}^{\dagger} c_{\beta_{2}} B_{\alpha_{2} \beta_{2} \alpha_{1} \beta_{1}} .
$$

To summarize, we have found that the partial trace density matrix $\rho \equiv \rho^{e}$ evolves according to

$$
\begin{aligned}
\partial_{t} \rho & =\mathbb{L}_{\lambda} \rho \\
& =\left(\mathbb{L}^{\text {free }}+\lambda^{2} \mathbb{L}^{\text {scatt }}\right) \rho,
\end{aligned}
$$

where

$$
\mathbb{L}^{\text {free }} \rho=-i\left[H_{e}, \rho\right]
$$

is the free evolution superoperator, and the conventional markovian superoperator $\mathbb{L}^{\text {scatt }}$ is given by

$$
\begin{aligned}
\mathbb{L}^{\text {scatt } \rho=} & -B_{\alpha_{1} \beta_{1} \alpha_{2} \beta_{2}} c_{\alpha_{1}}^{\dagger} c_{\beta_{1}} c_{\alpha_{2}}^{\dagger} c_{\beta_{2}} \rho \\
& -C_{\alpha_{1} \beta_{1} \alpha_{2} \beta_{2}} \rho c_{\alpha_{1}}^{\dagger} c_{\beta_{1}} c_{\alpha_{2}}^{\dagger} c_{\beta_{2}} \\
& +(B+C)_{\alpha_{2} \beta_{2} \alpha_{1} \beta_{1}} c_{\alpha_{1}}^{\dagger} c_{\beta_{1}} \rho c_{\alpha_{2}}^{\dagger} c_{\beta_{2}} .
\end{aligned}
$$

\subsection{One particle sector}

Since $\mathbb{L}^{\text {scatt }}$ in (46) commutes with the number operator, the subsystem number of particles is a constant of the motion. That means that if at time $t=0$ the initial density matrix is nonzero only in, say, the $n$-particle sector, then the evolution of $\rho$ according to equation (46) will keep $\rho(t)$ in that sector.

We are thus in position to examine the 1-particle sector. We will do so by writing (46) between one particle eigenstates $\left|\gamma_{1}\right\rangle$ and $\left|\gamma_{2}\right\rangle$.

Dropping the free evolution for sake of space, we compute

$$
\begin{aligned}
\partial_{t} \rho_{\gamma_{1} \gamma_{2}}= & -B_{\alpha_{1} \beta_{1} \alpha_{2} \beta_{2}} \delta_{\gamma_{1} \alpha_{1}} \delta_{\beta_{1} \alpha_{2}} \delta_{\beta_{2} \gamma_{1}^{\prime}} \rho_{\gamma_{1}^{\prime} \gamma_{2}^{\prime}} \delta_{\gamma_{2}^{\prime} \gamma_{2}} \\
& -\delta_{\gamma_{1} \gamma_{1}^{\prime}} \rho_{\gamma_{1}^{\prime} \gamma_{2}^{\prime}} \delta_{\gamma_{2}^{\prime} \alpha_{1}} \delta_{\beta_{1} \alpha_{2}} \delta_{\beta_{2} \gamma_{2}} C_{\alpha_{1} \beta_{1} \alpha_{2} \beta_{2}} \\
+ & (B+C)_{\alpha_{2} \beta_{2} \alpha_{1} \beta_{1}} \delta_{\gamma_{1} \alpha_{1}} \delta_{\beta_{1} \gamma_{1}^{\prime}} \rho_{\gamma_{1}^{\prime} \gamma_{2}^{\prime}} \delta_{\gamma_{2}^{\prime} \alpha_{2}} \delta_{\beta_{2} \gamma_{2}}
\end{aligned}
$$

which gives

$$
\begin{aligned}
\partial_{t} \rho_{\gamma_{1} \gamma_{2}}= & -B_{\gamma_{1} \gamma^{\prime \prime} \gamma^{\prime \prime} \gamma_{1}^{\prime}} \rho_{\gamma_{1}^{\prime} \gamma_{2}^{\prime}} \delta_{\gamma_{2}^{\prime} \gamma_{2}}-\delta_{\gamma_{1} \gamma_{1}^{\prime}} \rho_{\gamma_{1}^{\prime} \gamma_{2}^{\prime}} C_{\gamma_{2}^{\prime} \gamma^{\prime \prime} \gamma^{\prime \prime} \gamma_{2}} \\
& +(B+C)_{\gamma_{2}^{\prime} \gamma_{2} \gamma_{1} \gamma_{1}^{\prime}} \rho_{\gamma_{1}^{\prime} \gamma_{2}^{\prime}} .
\end{aligned}
$$

Switch back our notation to $\rho_{\alpha_{1} \alpha_{2}}$, we define the in- and out- one-particle scattering superoperators ${ }^{(1)} \Gamma^{\text {in/out }}$ by means of

$$
\partial_{t} \rho_{\alpha_{1} \alpha_{2}}=\left({ }^{(1)} \Gamma_{\alpha_{1} \alpha_{2}, \alpha_{1}^{\prime} \alpha_{2}^{\prime}}^{\mathrm{in}}{ }^{(1)} \Gamma_{\alpha_{1} \alpha_{2}, \alpha_{1}^{\prime} \alpha_{2}^{\prime}}^{\mathrm{out}}\right) \rho_{\alpha_{1}^{\prime} \alpha_{2}^{\prime}} .
$$

According to above, we find

$$
\begin{aligned}
& \frac{1}{2 \pi}{ }^{(1)} \Gamma_{\alpha_{1} \alpha_{2}, \alpha_{1}^{\prime} \alpha_{2}^{\prime}}^{\text {out }}=B_{\alpha_{1} \alpha^{\prime \prime} \alpha^{\prime \prime} \alpha_{1}^{\prime}} \delta_{\alpha_{2}^{\prime} \alpha_{2}}+\delta_{\alpha_{1} \alpha_{1}^{\prime}} C_{\alpha_{2}^{\prime} \alpha^{\prime \prime} \alpha^{\prime \prime} \alpha_{2}} \\
& \frac{1}{2 \pi}{ }^{(1)} \Gamma_{\alpha_{1} \alpha_{2}, \alpha_{1}^{\prime} \alpha_{2}^{\prime}}^{\text {in }}=(B+C)_{\alpha_{2}^{\prime} \alpha_{2} \alpha_{1} \alpha_{1}^{\prime}}
\end{aligned}
$$

\subsection{Single particle in the low density limit}

We now want to pass to the equation for the so called single-particle density matrix, which is the two-point Green function defined by

$$
\rho_{\alpha \alpha^{\prime}}^{\mathrm{sp}}=\left\langle c_{\alpha^{\prime}}^{\dagger} c_{\alpha}\right\rangle=\operatorname{Tr}\left(c_{\alpha} \rho c_{\alpha^{\prime}}^{\dagger}\right)
$$

Since the trace can be performed by summing over all the many body Hamiltonian eigenvalues, we note that

$$
\rho_{\alpha \alpha^{\prime}}^{\mathrm{sp}}=\left\langle 0\left|c_{\alpha} \rho c_{\alpha^{\prime}}^{\dagger}\right| 0\right\rangle+\sum_{\{n\} \mid \sum n_{j} \neq 0}\left\langle\{n\}\left|c_{\alpha} \rho c_{\alpha^{\prime}}^{\dagger}\right|\{n\}\right\rangle .
$$

The low density limit amounts to neglect two ore more particles content of the subsystem density matrix. In this limit, we drop the summation above to find

$$
\rho_{\alpha \alpha^{\prime}}^{\mathrm{sp}}=\left\langle\alpha|\rho| \alpha^{\prime}\right\rangle=\rho_{\alpha \alpha^{\prime}} .
$$

Due to this identity, the equation for the single particle density matrix is given exactly by the one-particle scattering operators, that is,

$$
\Gamma_{\alpha_{1} \alpha_{2}, \alpha_{1}^{\prime} \alpha_{2}^{\prime}}^{\mathrm{in} / \text { out }}={ }^{(1)} \Gamma_{\alpha_{1} \alpha_{2}, \alpha_{1}^{\prime} \alpha_{2}^{\prime}}^{\text {in }}
$$


To better compare with [32], we exploit the exchange properties $\mathcal{D}_{\alpha \beta, q}^{ \pm *}=\mathcal{D}_{\beta \alpha, q}^{\mp}$ and write

$$
\begin{aligned}
\frac{1}{2 \pi} \Gamma_{\alpha_{1} \alpha_{2}, \alpha_{1}^{\prime} \alpha_{2}^{\prime}}^{\mathrm{in}}= & g_{\alpha_{2}^{\prime} \alpha_{2}} g_{\alpha_{1}^{\prime} \alpha_{1}}^{*}(N+1)\left(\mathcal{D}_{\alpha_{1} \alpha_{1}^{\prime}}^{+}+\mathcal{D}_{\alpha_{2} \alpha_{2}^{\prime}}^{+*}\right) \\
& +g_{\alpha_{2} \alpha_{2}^{\prime}}^{*} g_{\alpha_{1} \alpha_{1}^{\prime}} N\left(\mathcal{D}_{\alpha_{1} \alpha_{1}^{\prime}}^{-}+\mathcal{D}_{\alpha_{2} \alpha_{2}^{\prime}}^{-*}\right),(55)
\end{aligned}
$$

and

$$
\begin{aligned}
& \frac{1}{2 \pi} \Gamma_{\alpha_{1} \alpha_{2}, \alpha_{1}^{\prime} \alpha_{2}^{\prime}}^{\text {out }}= \\
& \left(g_{\alpha_{1} \alpha^{\prime \prime}} g_{\alpha_{1}^{\prime} \alpha^{\prime \prime}}^{*}(N+1) \mathcal{D}_{\alpha^{\prime \prime} \alpha_{1}^{\prime}}^{+}+g_{\alpha^{\prime \prime} \alpha_{1}}^{*} g_{\alpha^{\prime \prime} \alpha_{1}^{\prime}} N \mathcal{D}_{\alpha^{\prime \prime} \alpha_{1}^{\prime}}^{-}\right) \delta_{\alpha_{2}^{\prime} \alpha_{2}} \\
& +\delta_{\alpha_{1} \alpha_{1}^{\prime}}\left(g_{\alpha_{2}^{\prime} \alpha^{\prime \prime}} g_{\alpha_{2} \alpha^{\prime \prime}}^{*}(N+1) \mathcal{D}_{\alpha^{\prime \prime} \alpha_{2}^{\prime}}^{+*}+g_{\alpha^{\prime \prime} \alpha_{2}^{\prime}}^{*} g_{\alpha^{\prime \prime} \alpha_{2}} N \mathcal{D}_{\alpha^{\prime \prime} \alpha_{2}^{\prime}}^{-*}\right)
\end{aligned}
$$

Thus, this is the general form of the CM superoperator for a quantum dot interacting with a thermal bosonic bath, in the low density limit. We just note that in its final form, for short, integration on the bath wavevectors $\mathbf{q}$ is understood.

\subsection{One phonon limit}

As it is, the CM superoperator we have obtained in the low density limit is still rather complex to analyze, due to the fact that, in general, one has to take into account contributions coming from each of the bath degrees of freedom q (which clearly form a continuum). Here we are going to examine its detailed structure by means of analytical methods: arguing on the non-locality of a typical electron-phonon interaction for a quantum dot, due to strong inhomogeneity, we shall see how our analysis could be restricted to the far easier context where all the bath contributions are concentrated around only one degree of freedom/wavevector. Due to its simplicity, this will allow us to better localize and understand possible intrinsic failures of the CM approach.

By inspecting equations (55) and (56), we readily see that the basic building block to construct the CM superoperator is given by the following integral

$$
I_{m}^{ \pm}(\underline{\alpha}, \underline{\beta})=\int_{\mathbb{R}^{3}} d^{3} \mathbf{q} f_{m}(\mathbf{q}) \mathcal{D}_{\alpha \beta, \mathbf{q}}^{ \pm}
$$

where for short $(\underline{\alpha}, \beta)=\left(\alpha, \alpha_{1}, \alpha_{2}, \beta, \beta_{1}, \beta_{2}\right)$, and we have put

$$
f_{m}(\mathbf{q})=g_{\alpha_{1} \beta_{1}}(\mathbf{q}) g_{\beta_{2} \alpha_{2}}(\mathbf{q})^{*}\left(N_{\omega_{b}(\mathbf{q})}+m\right)
$$

where $m=0,1$ labels emission and absorption terms.

Accordingly, considering equation (39), and performing some straightforward calculus, we see that

$$
I_{m}^{ \pm}(\underline{\alpha}, \underline{\beta})=I_{m}^{s, \pm}(\underline{\alpha}, \underline{\beta})+I_{m}^{r, \pm}(\underline{\alpha}, \underline{\beta})
$$

is the sum of a scattering part

$$
I_{m}^{s, \pm}(\underline{\alpha}, \underline{\beta})=\frac{1}{2} \int_{\Phi\left( \pm \omega_{\beta \alpha}\right)}^{d^{2} \mathbf{q}} \frac{f_{m}(\mathbf{q})}{\left\|\nabla \omega_{b}(\mathbf{q})\right\|}
$$

and an energy renormalisation part

$$
I_{m}^{r, \pm}(\underline{\alpha}, \underline{\beta})= \pm \mathcal{P} \int \frac{d \omega}{2 \pi i \omega} \int_{\Phi\left(\omega \pm \omega_{\beta \alpha}\right)} d^{2} \mathbf{q} \frac{f_{m}(\mathbf{q})}{\left\|\nabla \omega_{b}(\mathbf{q})\right\|} .
$$

In the above,

$$
\Phi(\omega)=\left\{\mathbf{q} \in \mathbb{R}^{3} \mid \omega_{b}(\mathbf{q})=\omega\right\}
$$

the (2-dimensional) $\mathbb{R}^{3}$-submanifold of phonons $\mathbf{q}$ that share the same energy $\omega$ (note however that $\Phi(\omega)$ could well be the empty set, for example when $\omega<0)$.

In general, the integral in equation (57) is a rather complex object to evaluate. As it involves an integration over all the space of the modes $\mathbf{q}$ (the momentum space), a first hint on how to evaluate it could come from trying to partition the momentum space in small cells, and then summing the contributions coming from each cell. Here we immediately face the question: to what extent are these cells independent?

As clearly pointed out in [32], a typical set of coupling parameters $g_{\alpha \beta}(\mathbf{q})$ satisfies

$$
g_{\alpha \beta}(-\mathbf{q})=g_{\beta \alpha}(\mathbf{q})^{*} .
$$

This in turn comes from the condition that the carrierphonon interaction Hamiltonian is assumed to be spatially local in the fields:

$$
H^{\prime}=\int d r \Psi^{\dagger}(r)\left(\phi^{\dagger}(r)+\phi(r)\right) \Psi(r)
$$

where integration is on all space, $\Psi(r)(\phi(r))$ destroys a carrier (respectively, a phonon) in the spatial point $r$.

If equation (63) held true, two different cells, centered at opposite momenta, would not be independent, and should be considered all together, but due to strong spatial inhomogeneity and anisotropy, equation (64) need not be valid any longer for the case of a quantum dot, so that the coupling parameters $g_{\alpha \beta}(\mathbf{q})$ do not suffer from the restriction (63) anymore.

This in turn means that the coupling parameters within each cell become independent, and to compute integral (57) we could safely partition the momentum space into cells, evaluating one at a time. Now the key point here is that the CM superoperator depends linearly on each single cell contribution, and the precise relationship among different cells only depends on the details of the carrier-phonon interaction, as well as on the properties of the phonon bath, but is not to any extent depending on the intrinsic structure of the CM superoperator itself. In order to understand properties which are intrinsic to the CM superoperator, it is therefore convenient to analyze the contribution coming from one single cell, by claiming that possible anomalies need not be recovered by counterbalancing contributions from other cells. Whether those other contributions succeed in counter-balancing pathologies arising from the original cell, or not, strongly depends on the details of the model, and therefore does not happen generally.

With respect to this one could say that for the large class of models for which the interaction Hamiltonian is 
of the form (64) one should consider cells at opposite momenta at the same time, and this fact could eliminate pathologies. Within the same spirit of our argument above, we answer that the Hamiltonian in (64) is not intrinsic to the CM approach, and thus should not be taken as a reference; moreover, pathologies would indeed remain, but to show why and how would take a too long technical procedure, due to the need of evaluating opposite cells at the same time, and thus goes out of the scope of the present paper.

We shall therefore proceed to compute equation (57) in case the coupling parameters be nonzero only inside a cell in the momentum space. The cell will be taken small enough so that quantities like the bath dispersion don't vary too rapidly within it, thus allowing for an actual analytical evaluation of equation (57) in what we call the "one-phonon" limit. Again, with this terminology we want to focus on a specific and small enough cell in the momentum space of the bath: thus, we will always consider an interaction of the system (the quantum dot) with a continuum of bath modes, and by no means we are going to reduce the number of interacting phonon modes to just one, or to a discrete number. This will be so because we will scale the couplings at the same time as we do the limit of small cell, so as to keep information about an interaction with a continuum of modes, rather than with a discrete spectrum in the bath. The latter case indeed describes an oscillating behavior, with no possible damping. Within this philosophy, contrary to optical phonons, that call for a polaronic description in quantum dots [36], we shall consider the case of acoustic phonons, for which there always exists a phonon mode $\mathbf{q}$ that guarantees energy conservation.

To be more precise, let us fix a reference phonon mode $\overline{\mathbf{q}}$, at the center of the cell, given in spherical coordinates by $\overline{\mathbf{q}}=(\bar{R}, \bar{\theta}, \bar{\phi})$. We furthermore suppose that the phonon mode $\overline{\mathbf{q}}$ is resonant with some specific subsystem transition, namely, $\omega_{b}(\overline{\mathbf{q}})=\Delta \omega=\omega_{\alpha \beta}$ for some nonzero $\omega_{\alpha \beta} \equiv \omega_{\alpha}-\omega_{\beta}$. This hypothesis is of course needed in order to obtain a nonzero scattering contribution for our CM superoperator. We then choose the cell according to the polar sector

$$
\begin{aligned}
R & \in\left[\bar{R}-\frac{\Delta R}{2}, \bar{R}+\frac{\Delta R}{2}\right] \\
\theta & \in\left[\bar{\theta}-\frac{\Delta \theta}{2}, \bar{\theta}+\frac{\Delta \theta}{2}\right] \\
\phi & \in\left[\bar{\phi}-\frac{\Delta \phi}{2}, \bar{\phi}+\frac{\Delta \phi}{2}\right]
\end{aligned}
$$

where we take $\Delta R$ small enough, so that no mode in the polar sector is resonant with a transition different from $\Delta \omega$. We let $\chi(\mathbf{q})$ be the characteristic function of the polar set we have just defined (which by definition takes value 1 inside the cell above, and 0 outside). Note that the volume of the polar sector is given by

$$
V=\int d^{3} \mathbf{q} \chi(\mathbf{q})=\bar{R}^{2} \Delta R \Delta \phi \int_{\bar{\theta}-\frac{\Delta \theta}{2}}^{\bar{\theta}+\frac{\Delta \theta}{2}} d \theta \sin \theta
$$

We define our coupling parameters as

$$
g_{\alpha \beta}(\mathbf{q})=\chi(\mathbf{q}) \frac{1}{\sqrt{V}} h_{\alpha \beta}
$$

for (q independent) coupling frequencies $h_{\alpha \beta}$ (note indeed that $h_{\alpha \beta}$ are measured in $\mathrm{Hz}$ ).

Moreover, we suppose the phonon dispersion law in the polar sector above to be sufficiently well behaved, in the sense that

$$
\omega_{b}(\mathbf{q})=\omega_{0}+\nabla \omega_{b}(\overline{\mathbf{q}}) \cdot \mathbf{q}+o(\Delta R)
$$

for every phonon mode $\mathbf{q}$ in the cell.

Now of course one would like to evaluate equation (59), to first order in $\Delta R$. Considering the latter equation, splitting our total integral into a scattering and a renormalization part, we see that keeping a finite limit for the scattering integral (60) forces the renormalization part (61) to disappear, unless some particular scaling of the dispersion gradient $\nabla \omega_{b}(\overline{\mathbf{q}})$ is assumed (we shall however keep the latter quantity always finite, as it is the case for acoustic phonons). This is so because the renormalization integral amounts to an integration, on the interval $\pm \Delta R / 2$, of quantities that are of the same order of magnitude as in the scattering integral. Therefore, in our "one-phonon" limit, it suffices to consider the scattering part, which is computed to be

$$
\begin{aligned}
I_{m}^{s, \pm}(\underline{\alpha}, \underline{\beta})= & \frac{1}{2} \delta_{\Delta \omega, \pm \omega_{\beta \alpha}} \frac{h_{\alpha_{1} \beta_{1}} h_{\beta_{2} \alpha_{2}}^{*}}{\mid \nabla \omega_{b}(\overline{\mathbf{q}})}(N+m) \\
& \times \int d \theta d \phi \frac{\bar{R}^{2}}{V} \sin \theta \chi(\mathbf{q}) \\
= & \frac{1}{2} \delta_{\Delta \omega, \pm \omega_{\beta \alpha}} \frac{h_{\alpha_{1} \beta_{1}} h_{\beta_{2} \alpha_{2}}^{*}}{\left|\nabla \omega_{b}(\overline{\mathbf{q}})\right| \Delta R}(N+m) .
\end{aligned}
$$

Note that the Kroenecher delta has appeared not because of the Dirac energy conserving delta function (which is automatically taken into account by the surface integral), but rather, it is due to our one-phonon approximation: we have chosen $\Delta R$ so small, that $\Phi\left( \pm \omega_{\beta \alpha}\right)=\emptyset$ is the empty set whenever $\pm \omega_{\beta \alpha} \neq \Delta \omega$. We would also like to note, in passing, that we have performed the substitution $R \rightarrow \bar{R}$ in the surface integral above, because of our hypothesis on the bath dispersion in our cell.

Now, scaling the coupling frequencies

$$
h_{\alpha \beta}=\sqrt{\Delta \omega\left|\nabla \omega_{b}(\overline{\mathbf{q}})\right| \Delta R} a_{\alpha \beta}
$$

through dimensionless (complex) quantities $a_{\alpha \beta}$, allows us to conclude that the integral in (57) boils down to

$$
\begin{array}{r}
\int d^{3} \mathbf{q} 2 \pi\left(\frac{1}{2} \pm \frac{1}{2}+N_{\mathbf{q}}\right) g_{\alpha_{1} \alpha_{1}^{\prime}}(\mathbf{q})^{*} g_{\beta_{1} \beta_{1}^{\prime}}(\mathbf{q}) \mathcal{D}_{\alpha \beta, \mathbf{q}}^{\mathrm{c}-\mathrm{qp}, \pm} \\
\rightarrow \pi\left(\frac{1}{2} \pm \frac{1}{2}+N\right) a_{\alpha_{1} \alpha_{1}^{\prime}}^{*} a_{\beta_{1} \beta_{1}^{\prime}} \Delta \omega \delta_{\Delta \omega, \pm \omega_{\beta \alpha}}
\end{array}
$$

in the "one-phonon" limit. The result above indicates that the one-phonon limit is equivalent to 
- consider a bath described by exactly one phonon, of energy $\Delta \omega$, to which the system is resonantly coupled by means of coupling energies $\Delta \omega a_{\alpha \beta}$;

- disregard energy renormalisation effects;

- substitute the Dirac delta distribution with

$$
\delta\left(\Delta \omega \pm \omega_{\alpha \beta}\right) \rightarrow(\Delta \omega)^{-1} \delta_{\Delta \omega, \pm \omega_{\beta \alpha}},
$$

where $\delta$ in the right hand side is the Kronecker delta.

Again, this procedure is only formal, and physically corresponds to adopt the above scalings to evaluate an interaction with a whole bunch of phonon modes, which form a continuum, even if localized in a finite cell.

Now dimensional analysis suggests that the quantity

$$
\begin{aligned}
\Upsilon & =\frac{V}{\omega_{b}(\overline{\mathbf{q}}) \Delta R} \sum_{\alpha \beta}\left|g_{\alpha \beta}(\overline{\mathbf{q}})\right|^{2} \\
& =\frac{\bar{R}^{2} \sin \bar{\theta} \Delta \theta \Delta \phi}{\omega_{b}(\overline{\mathbf{q}})} \sum_{\alpha \beta}\left|g_{\alpha \beta}(\overline{\mathbf{q}})\right|^{2}
\end{aligned}
$$

could be taken to represent a measure of how big the perturbation $H^{\prime}$ is (indeed, it is measured in $\mathrm{Hz}$, as can be seen).

Accordingly, the weak-coupling regime is reached when this quantity is of the same order of the system transitions $\Delta \omega$, and $|\lambda| \ll 1$.

Then, in terms of the adimensional coupling parameters $a_{\alpha \beta}$, the weak-coupling condition above is restated as

$$
\frac{\Upsilon}{\Delta \omega}=\sum_{\alpha \beta}\left|a_{\alpha \beta}\right|^{2} \approx 1, \quad \lambda \ll 1 .
$$

\subsection{Explicit form of the CM superoperator in the "one-phonon" limit}

Using equation (71), we write our scattering operators in the one-phonon limit:

$$
\begin{aligned}
& \frac{1}{\pi \Delta \omega} \Gamma_{\alpha_{1} \alpha_{2}, \alpha_{1}^{\prime} \alpha_{2}^{\prime}}^{\mathrm{in}}=a_{\alpha_{2}^{\prime} \alpha_{2}} a_{\alpha_{1}^{\prime} \alpha_{1}}^{*}(N+1)\left(\delta_{\Delta \omega,-\omega_{\alpha_{1} \alpha_{1}^{\prime}}}\right. \\
& \left.\quad+\delta_{\Delta \omega,-\omega_{\alpha_{2} \alpha_{2}^{\prime}}}\right)+a_{\alpha_{2} \alpha_{2}^{\prime}}^{*} a_{\alpha_{1} \alpha_{1}^{\prime}} N\left(\delta_{\Delta \omega, \omega_{\alpha_{1} \alpha_{1}^{\prime}}}+\delta_{\Delta \omega, \omega_{\alpha_{2} \alpha_{2}^{\prime}}}\right) .
\end{aligned}
$$

and

$$
\begin{array}{r}
\frac{1}{\pi \Delta \omega} \Gamma_{\alpha_{1} \alpha_{2}, \alpha_{1}^{\prime} \alpha_{2}^{\prime}}^{\text {out }}=\left(a_{\alpha_{1} \alpha^{\prime \prime}} a_{\alpha_{1}^{\prime} \alpha^{\prime \prime}}^{*}(N+1) \delta_{\Delta \omega,-\omega_{\alpha^{\prime \prime} \alpha_{1}^{\prime}}}\right. \\
\left.+a_{\alpha^{\prime \prime} \alpha_{1}}^{*} a_{\alpha^{\prime \prime} \alpha_{1}^{\prime}} N \delta_{\Delta \omega, \omega_{\alpha^{\prime \prime} \alpha_{1}^{\prime}}}\right) \delta_{\alpha_{2}^{\prime} \alpha_{2}} \\
+\delta_{\alpha_{1} \alpha_{1}^{\prime}}\left(a_{\alpha_{2}^{\prime} \alpha^{\prime \prime}} a_{\alpha_{2} \alpha^{\prime \prime}}^{*}(N+1) \delta_{\Delta \omega,-\omega_{\alpha^{\prime \prime} \alpha_{2}^{\prime}}}\right. \\
\left.+a_{\alpha^{\prime \prime} \alpha_{2}^{\prime}}^{*} a_{\alpha^{\prime \prime} \alpha_{2}} N \delta_{\Delta \omega, \omega_{\alpha^{\prime \prime} \alpha_{2}^{\prime}}}\right) .
\end{array}
$$

To proceed, it's clear that $\alpha \rightarrow \beta$ transitions are allowed only if $\omega_{\alpha \beta}= \pm \Delta \omega$, so it is no loss of generality to assume an $n$-level system, with levels labeled by $\alpha=1 \ldots n$, of equally spaced energies

$$
\omega_{\alpha}=(\alpha-1) \Delta \omega
$$

so that $\omega_{1}=0, \omega_{2}=\Delta \omega, \ldots, \omega_{n}=(n-1) \Delta \omega$ in increasing order.

To write the superoperator in matrix form, we introduce the matrix-to-vector mapping $\rho \mapsto \widetilde{\rho}$ given by

$$
\widetilde{\rho}_{i}=\sum_{\alpha \beta} \rho_{\alpha \beta} \delta_{i,(\alpha-1) n+\beta},
$$

valid for all $n$. For example, for $n=2$ we have $\widetilde{\rho}_{1}=$ $\rho_{11}, \widetilde{\rho}_{2}=\rho_{12}, \widetilde{\rho}_{3}=\rho_{21}, \widetilde{\rho}_{4}=\rho_{22}$. It follows that the matrix representation $\widetilde{\mathbb{L}}$ for a generic superoperator $\mathbb{L}$ is defined by

$$
\widetilde{\mathbb{L}} \widetilde{\rho}:=\widetilde{\mathbb{L} \rho}, \quad \forall \rho .
$$

For example, in case $n=2$, we obtain the following matrix representation $\widetilde{\mathbb{L}}$ in terms of the coefficients $\mathbb{L}_{\alpha_{1} \alpha_{2}, \alpha_{1}^{\prime} \alpha_{2}^{\prime}}$ of the superoperator $\mathbb{L}$ :

$$
\widetilde{\mathbb{L}}=\left(\begin{array}{llll}
\mathbb{L}_{11,11} & \mathbb{L}_{11,12} & \mathbb{L}_{11,21} & \mathbb{L}_{11,22} \\
\mathbb{L}_{12,11} & \mathbb{L}_{12,12} & \mathbb{L}_{12,21} & \mathbb{L}_{12,22} \\
\mathbb{L}_{21,11} & \mathbb{L}_{21,12} & \mathbb{L}_{21,21} & \mathbb{L}_{21,22} \\
\mathbb{L}_{22,11} & \mathbb{L}_{22,12} & \mathbb{L}_{22,21} & \mathbb{L}_{22,22}
\end{array}\right) .
$$

\subsection{2-level system}

We now study the case $n=2$ (a two level system). Accordingly, we obtain

$$
\frac{d \widetilde{\rho}}{d t}=\widetilde{\mathbb{L}}_{\lambda} \widetilde{\rho}
$$

with $\widetilde{\mathbb{L}}_{\lambda}=\widetilde{\mathbb{L}}^{\text {free }}+\lambda^{2} \widetilde{\mathbb{L}}^{\text {scatt }}$. We compute the (matrix representation of the) free generator to be

$$
\widetilde{\mathbb{L}}^{\text {free }}=\Delta \omega\left(\begin{array}{cccc}
0 & 0 & 0 & 0 \\
0 & i & 0 & 0 \\
0 & 0 & -i & 0 \\
0 & 0 & 0 & 0
\end{array}\right) .
$$

For the scattering part, we define the following dimensionless variables

$$
\begin{aligned}
& \zeta=a_{21}^{*} \frac{a_{11}-a_{22}}{\left|a_{21}\right|^{2}} \\
& \eta=\frac{a_{12}}{a_{21}}
\end{aligned}
$$

and find

$$
\begin{aligned}
\widetilde{\mathbb{L}}^{\text {scatt }} & =2 \pi \Delta \omega\left|a_{21}\right|^{2} \\
& \times\left(\begin{array}{llll}
-N & 0 & 0 & N+1 \\
\zeta \frac{N}{2} & -\frac{2 N+1}{2} & \eta \frac{2 N+1}{2} & -\zeta \frac{N+1}{2} \\
\zeta^{*} \frac{N}{2} & \eta^{*} \frac{2 N+1}{2} & -\frac{2 N+1}{2} & -\zeta^{*} \frac{N+1}{2} \\
N & 0 & 0 & -(N+1)
\end{array}\right) .
\end{aligned}
$$


In passing, it is instructive to note that the superoperator just found disappears in case $\left|a_{21}\right|=\left|a_{12}\right|=0$, that is, in case of diagonal coupling. This means that the decay process due to diagonal coupling (pure dephasing) cannot be described in markovian fashion.

Now, putting

$$
\begin{aligned}
\xi & =i \zeta \\
\epsilon & =2 \pi \hbar \Delta \omega\left|a_{21}\right|^{2} \\
& =2 \pi \hbar\left(\frac{\partial \omega_{b}(\bar{q})}{\partial V}\right)^{-1}\left|g_{21}\right|^{2}
\end{aligned}
$$

we find agreement with Ref. [32] in the particular case of $\eta=1$ and $\xi$ being a real number: indeed, we obtain

$$
\widetilde{\mathbb{L}}^{\text {scatt }}=\frac{\epsilon}{\hbar}\left(\begin{array}{llll}
-N & 0 & 0 & N+1 \\
-i \xi \frac{N}{2} & -\frac{2 N+1}{2} & \frac{2 N+1}{2} & i \xi \frac{N+1}{2} \\
i \xi \frac{N}{2} & \frac{2 N+1}{2} & -\frac{2 N+1}{2} & -i \xi \frac{N+1}{2} \\
N & 0 & 0 & -(N+1)
\end{array}\right) .
$$

This is exactly equation (125) in reference [32], up to suitable row and column flips, due to the different ordering procedure chosen in the matrix representation $\widetilde{\mathbb{L}}$ of the scattering superoperators $\mathbb{L}$.

Now it can be checked that the thermal solution

$$
\rho_{\mathrm{T}}=\left(\begin{array}{cc}
\frac{N+1}{2 N+1} & 0 \\
0 & \frac{N}{2 N+1}
\end{array}\right),
$$

corresponding to the vector

$$
\widetilde{\rho}_{\mathrm{T}}=\frac{1}{2 N+1}(N+1,0,0, N),
$$

is a steady state for $\widetilde{\mathbb{L}}^{\text {scatt }}$, and thus also for $\widetilde{\mathbb{L}}_{\lambda}=\widetilde{\mathbb{L}}^{\text {free }}+$ $\lambda^{2} \widetilde{\mathbb{L}}^{\text {scatt }}$. That is, by inspection one realizes that $\widetilde{\mathbb{L}}_{\lambda} \widetilde{\rho}_{\mathrm{T}}=$ 0 , and so

$$
\mathbb{L}_{\lambda} \rho_{\mathrm{T}}=0
$$

independently of the chosen matrix representation, and for all values of the coupling constant $\lambda$, of the occupation number $N$, and even for arbitrary complex coupling parameters $a_{\alpha \alpha^{\prime}}$. This by itself is undoubtedly a good feature of the CM superoperator. But can the thermal density matrix be ever reached?

\section{Steady state stability}

Once proven that the thermal density matrix is a steady state solution, we are now going to address the problem of stability of such a solution. For this, we will write down the other three remaining eigenvalues of $\widetilde{\mathbb{L}}_{\lambda}$ (the first found being eig $\left._{1}=0\right)$ : introducing, also for later convenience, the dimensionless constants

$$
\begin{aligned}
\kappa & =2 \pi\left|a_{21}\right|^{2}(2 N+1)=\frac{\epsilon}{\hbar \Delta \omega}(2 N+1) \\
\kappa^{\prime} & =2 \pi\left|a_{12}\right|^{2}(2 N+1),
\end{aligned}
$$

they are found to be $\mathrm{eig}_{2}=-\lambda^{2} \kappa^{\prime} \Delta \omega$, and

$$
\operatorname{eig}_{3,4}=-\frac{\Delta \omega}{2}\left(\lambda^{2} \kappa^{\prime} \pm \sqrt{\lambda^{4} \kappa \kappa^{\prime}-4}\right)
$$

In particular, we see that the real part of eig $_{4}$ (the one associated to - above) becomes positive as soon as

$$
\lambda^{4} \kappa^{\prime}\left(\kappa-\kappa^{\prime}\right)>4
$$

Note that the latter inequality is never satisfied when $\kappa \leq \kappa^{\prime}$ (or $|\eta| \leq 1$, or $\left|a_{12}\right| \leq\left|a_{21}\right|$ ), whereas it can be satisfied in the opposite case $\kappa>\kappa^{\prime}$ (or $|\eta|>1$, or $\left.\left|a_{12}\right|>\left|a_{21}\right|\right)$, furnishing explicit spectral evidence of the lack of symmetry in the CM superoperator: coherent processes of the type $1 \rightarrow 2$ and $2 \rightarrow 1$ undergo different markovian approximations, within the CM scheme. We report in Figure 1 the real part of the spectrum of $\mathbb{L}_{\lambda}$, in units of $\Delta \omega$ and for different values of $|\eta|$, as a function of the dimensionless coupling constant $\lambda$.

In the following, we are going to analyze the precise origin, and meaning, of the above spectral condition, to see whether or not it could put at variance the very same consistency of the CM approximation scheme at long times.

We proceed to a direct inspection of

$$
\partial_{t} \rho=\mathbb{L}_{\lambda} \rho
$$

through the explicit and most general form of $\widetilde{\mathbb{L}}^{\text {scatt }}$ that we have computed in equation (84). We find a coupled system of equations for the elements of the density matrix, which we are now going to put in a useful form, where populations evolve independently, and polarizations evolve thanks to population and polarization terms. Let us therefore introduce the the $T_{1}$-time matrix

$$
A=\frac{1}{2 N+1}\left(\begin{array}{cc}
-N & N+1 \\
N & -N+1
\end{array}\right) .
$$

Then populations evolve according to

$$
\partial_{t}\left(\begin{array}{l}
\rho_{11} \\
\rho_{22}
\end{array}\right)=\lambda^{2} \Delta \omega \kappa A\left(\begin{array}{c}
\rho_{11} \\
\rho_{22}
\end{array}\right) .
$$

The eigenvalues of the $T_{1}$-times matrix $A$ are easily computed to be -1 and 0 ; the change of base matrix $P$ containing the respective normalized eigenvectors in its columns is defined by

$$
P=\left(\underline{e}_{-1}, \underline{e}_{0}\right)=\left(\begin{array}{cc}
-\frac{1}{2} & \frac{N+1}{2 N+1} \\
\frac{1}{2} & \frac{N}{2 N+1}
\end{array}\right)
$$

(note that the mode $\underline{e}_{0}$ relative to the zero eigenvalue is nothing but the thermal state). Then passing to eigenvariables $\underline{\Lambda}=\left(\Lambda_{1}, \Lambda_{2}\right)^{T}$ with

$$
\left(\begin{array}{l}
\rho_{11} \\
\rho_{22}
\end{array}\right)=P \underline{\Lambda}
$$

we solve the population sector with

$$
\begin{aligned}
& \partial_{t} \Lambda_{1}=-\lambda^{2} \Delta \omega \kappa \Lambda_{1} \\
& \partial_{t} \Lambda_{2}=0 .
\end{aligned}
$$




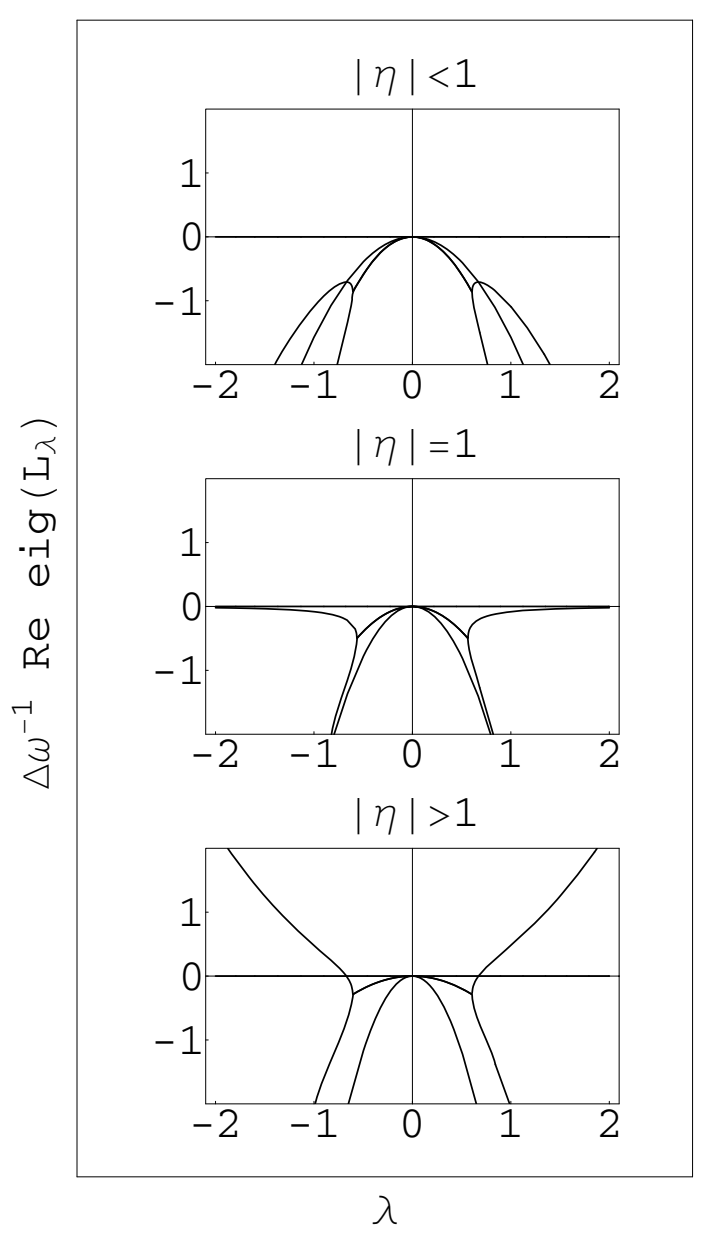

Fig. 1. Real part of $\mathbb{L}_{\lambda}$ eigenvalues in units of the phonon energy $\Delta \omega$, as a function of the dimensionless coupling constant $\lambda$. The chosen parameters correspond to room temperature conditions $T \sim 300{ }^{\circ} \mathrm{K}$, and weak coupling condition $\left|a_{12}\right|^{2}+\left|a_{21}\right|^{2}=1 / 2$ (see discussion on $\Upsilon$ in the text). In the first two graphs $(|\eta|=1 / \sqrt{3}$ and $|\eta|=1$ respectively) all eigenvalues have negative real parts, except the zero eigenvalue, associated to the thermal steady state. However, the zero eigenvalue tends to be degenerate in the second case, for large values of the coupling constant $\lambda$. In the third graph $|\eta|=\sqrt{3}$, and this asymptotic degeneracy is broken through a crossover of the horizontal axis, leading to unphysical divergences due to high instability of the steady state solution.

We define the dimensionless constant

$$
\sigma=\frac{\lambda^{2} \kappa}{2}
$$

and note that $2 \Delta \omega \sigma$ is the inverse $T_{1}$ time for the even mode $\Lambda_{1}$, that is, the population mode orthogonal to the thermal one. We proceed now to write down the equation for the polarization sector. We define the polarization vector

$$
\underline{\rho}=\left(\begin{array}{c}
\rho_{12} \\
\rho_{21}
\end{array}\right)
$$

and the $p-f$ (polarization to population) coupling vector

$$
\underline{\zeta}=\left(\begin{array}{c}
\zeta \\
\zeta^{*}
\end{array}\right) .
$$

Then we find

$$
\partial_{t} \underline{\rho}=\Delta \omega\left(B \underline{\rho}+\frac{\sigma}{2} \Lambda_{1} \underline{\zeta}\right)
$$

where

$$
B=\left(\begin{array}{cc}
i-\sigma & \sigma \eta \\
\sigma \eta^{*} & -i-\sigma
\end{array}\right)
$$

and we recall that the even mode $\Lambda_{1}$ is time dependent, and is evaluated at current time $t$. Since the nonhomogeneous source terms $-\frac{\lambda^{2} \gamma \zeta^{ \pm}}{4} \Lambda_{1}$ act as a relaxation source, divergences will show up if and only if the linear homogeneous part is divergent: here we are going to show that this is indeed what happens. Now calling $a=\sqrt{\sigma^{2}|\eta|^{2}-1}$ and $b=\sigma \eta$, we see that $B$ is diagonalized as $B=P C P^{-1}$ with

$$
C=\left(\begin{array}{cc}
\mathrm{eig}_{+} & 0 \\
0 & \text { eig }
\end{array}\right)=\left(\begin{array}{cc}
-\sigma+a & 0 \\
0 & -\sigma-a
\end{array}\right),
$$

by going to diagonalizing modes $\underline{\Pi}=\left(\Pi_{+}, \Pi_{-}\right)^{T}$ through $\underline{\rho}=P \underline{\Pi}$, thanks to the change of base matrix

$$
P=\left(\begin{array}{cc}
1 & \frac{i-a^{*}}{b^{*}} \\
\frac{a^{*}-i}{b} & 1
\end{array}\right)
$$

By inspecting the latter diagonalizing matrix $P$, we note that when $a$ is pure imaginary or zero (which corresponds to $\sigma|\eta| \leq 1$ ), then the polarization modes evolve coherently by always maintaining the reality condition $\Pi_{+}=\Pi_{-}^{*}$. This is not true anymore when $a$ becomes real, that is, when $\sigma|\eta|>1$ strictly. At that branching point, the latter reality condition is no more maintained, and the two polarization modes become independent variables (this is clearly visualized in Fig. 1). But pathologies occur when $a>\sigma$, that is when the real part of eigenvalue eig for $B$ becomes positive: then we have $a>0$ a fortiori. For this reason, from now on we shall put ourselves beyond the branching point, that is, we shall assume $a>0$. Then we can write the inverse for $P$ as

$$
P^{-1}=\frac{1}{2 a}\left(\begin{array}{cc}
\frac{|b|^{2}}{a-i} & b \\
-b^{*} & \frac{|b|^{2}}{a-i}
\end{array}\right),
$$

and it follows that we have completely decoupled our system with

$$
\partial_{t} \underline{\Pi}=\Delta \omega\left(C \underline{\Pi}+\frac{\sigma}{2} \Lambda_{1} P^{-1} \underline{\zeta}\right) .
$$

At this point it is important to note that

$$
\Lambda_{1}(t)=e^{-2 \sigma \Delta \omega t} \Lambda_{1}(0)
$$

goes to zero as $t \rightarrow+\infty$, as $\sigma>0$ (always). Then, since the eigenvector eig - $_{-}=-(\sigma+a)$ has negative real part (for $a>0$, as we are assuming), it follows that the associated 
polarization mode $\Pi_{-}$decays to zero when $t \rightarrow+\infty$. Then we obtain the following asymptotical condition, valid for large times $t \gg 0$ :

$$
\left(\begin{array}{c}
\rho_{12}(t) \\
\rho_{21}(t)
\end{array}\right) \stackrel{t \gg 0}{\sim} P\left(\begin{array}{c}
\Pi_{+}(t) \\
0
\end{array}\right)=\left(\begin{array}{c}
\Pi_{+}(t) \\
\Pi_{+}(t)^{*}
\end{array}\right),
$$

which indicates that, for large times, the polarization $\rho_{12}$ is asymptotic to the polarization mode $\Pi_{+}$. The last equality requires a bit of algebra: for example one can put $\left(X_{1}, X_{2}\right)^{T}=P\left(\Pi_{+}(t), 0\right)^{T}$, and then check that $\partial_{t} X_{2}(t)=$ $\partial_{t} \Pi_{+}(t)^{*}$.

Then we proceed to solve the equation for $\Pi_{+}$: although it can be solved exactly (as the one for $\Pi_{-}$), we put ourselves in the pathological situation eig ${ }_{+}=-\sigma+a>0$, and find the following long time asymptotic behavior

$$
\rho_{12}(t) \sim \Pi_{+}(t) \sim e^{(a-\sigma) \Delta \omega t}\left\{\Pi_{+}(0)+S \Lambda_{1}(0)\right\},
$$

valid for $t \gg 0$, with the source $S$ being

$$
S=\frac{\sigma}{4 a^{2}}\left(\frac{|b|^{2}}{a-i} \zeta+b \zeta^{*}\right)
$$

This last result is extremely important: it has been deduced when $a>\sigma$, that is when $\sigma^{2}\left(|\eta|^{2}-1\right)>1$, and it shows that the polarization terms $\rho_{12}(t)=\rho_{21}(t)^{*}$ diverge exponentially, as soon as the initial condition is chosen according to $\Pi(0) \neq 0$ or $\Lambda_{1}(0) \neq 0$. Indeed, we note that, in general, the source term is nonzero: it becomes zero only for the very particular choice of the coupling parameters given by

$$
\frac{\zeta}{\zeta^{*}}=\frac{i-a}{b^{*}}=\frac{i-\sqrt{\sigma^{2}|\eta|^{2}-1}}{\sigma \eta^{*}},
$$

which in fact produces complete destructive interference, and totally decouples the polarization mode $\Pi_{+}$from the population mode $\Lambda_{1}$. For every other configuration we obtain $S \neq 0$, and we see that it suffices to start with an arbitrarily small but nonzero perturbation of the thermal solution $\Lambda_{1}(0) \neq 0$, that, even when the polarizations are initially set to zero (implying $\Pi_{+}(0)=0$ ), the polarizations $\rho_{12}(t)$ generate out of the perturbation, and diverge. We stress that putting a (arbitrarily small) $\Lambda_{1}(0) \neq 0$, while keeping $\Pi_{+}(0)=0$, amounts to perform a diagonal perturbation, that only slightly shifts the population distribution with respect to the thermal solution, leaving the polarizations untouched, that is, set to zero.

We are now going to see that this behavior runs completely counter what should be expected, as divergences have growing velocities as the temperature is raised. The condition $a>\sigma$ can easily be seen to exactly match our original spectral condition on the eigenvalue eig $_{4}$ of the CM superoperator $\mathbb{L}_{\lambda}$, that is, it amounts to

$$
\lambda^{4} \kappa^{\prime}\left(\kappa-\kappa^{\prime}\right)>4
$$

As before, we stress that this condition is never satisfied when $|\eta| \leq 1$, but can be satisfied when $|\eta|>1$, explicitly showing the lack of symmetry of the CM approach. For $|\eta|>1$, it boils down to a condition on the temperature, through the occupation number $N$ :

$$
2 N+1>\frac{1}{\lambda^{2} \pi} \frac{1}{\left|a_{21}\right| \sqrt{\left|a_{12}\right|^{2}-\left|a_{21}\right|^{2}}} .
$$

It is easy to show that this inequality may well be satisfied within the weak-coupling regime: for example, since the weak-coupling limit requirement states that $\Upsilon / \Delta \omega=$ $\sum\left|a_{\alpha \beta}\right|^{2} \approx 1$, we take $\left|a_{12}\right|^{2}+\left|a_{21}\right|^{2}=c^{2}$, for some $0<c<1$, and see that the worst case (i.e. the lowest critical temperature) is reached when $|\eta|=\sqrt{3}$ (with $\left|a_{12}\right|=c \sqrt{3} / 2$ and $\left|a_{21}\right|=c / 2$ ), where it becomes

$$
2 N+1>\frac{2 \sqrt{2}}{\lambda^{2} \pi c^{2}} .
$$

This is a very unphysical behavior: we have found that, although the thermal solution is always a steady state, even the smallest polarization perturbation of the thermal solution will diverge beyond the above critical temperature, leading to non negligible negative eigenvectors for the density matrix within finite times. Indeed, it would be easy to further deduce that, asymptotically, the eigenvalues of the density matrix are given by $\pm\left|\rho_{12}(t)\right|$, as $t \rightarrow \infty$. But this shows a very important and non-negligible instability feature, which completely precludes the study of quantum decoherence and dissipation at asymptotically large times through the CM approach.

Completing the analysis for the initial transient is just a matter of a bit more patience: one also has to care about the other polarization mode, which exponentially damps, and also about invertibility of polarization diagonalizing matrix $P$, which physically corresponds to non-diverging coherent rotation, etc. We do not report the complete solution here, as the analytical results obtained so far largely suffice to our purposes. In Figure 2 we plot the time dependent density matrix elements, when the coupling parameters $a_{\alpha \beta}$ satisfy divergence/instability conditions, and the thermal distribution is perturbed by no more that $10^{-5}$. Results are in complete agreement with our present argument, and eigenvalue divergence of the evolving density matrix is observed. Physically, one could perhaps be sloppy and say that, normally, below a critical temperature, the system relaxes thermally, $\rho(t) \rightarrow \rho_{T}$; but above such a critical temperature (that scales with $\lambda^{-2}$ ), and when the coupling parameters satisfy $|\eta|>1$, the system relaxes to the unphysical result

$$
\rho_{\text {steady }}=\left(\begin{array}{cc}
\frac{N+1}{2 N+1} & \infty \\
\infty^{*} & \frac{N}{2 N+1}
\end{array}\right) .
$$

To conclude this section, we have shown very important intrinsic limitations of the CM approach when employed for large time or steady state analysis. Even neglecting the renormalisation part gives a good steady state (thermal), which however is highly unstable: arbitrarily small perturbations need not relax thermally back to the steady solution, and may well lead to divergent polarizations. Our results show that these intrinsic limitations already show 


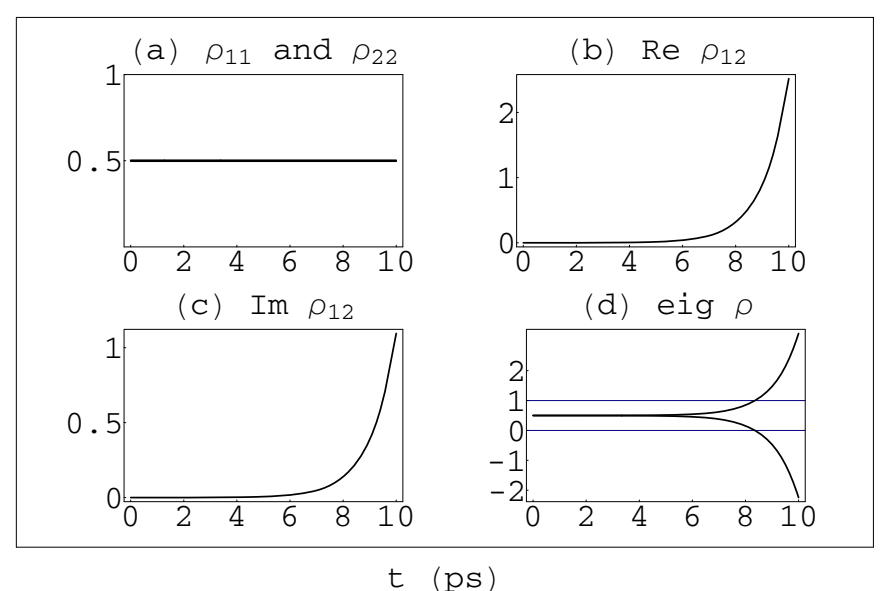

Fig. 2. Time evolution of the density matrix with initial conditions given by $\rho_{11}(0)=\left(1+10^{-5}\right) \frac{N+1}{2 N+1}, \rho_{22}(0)=1-\rho_{11}(0)$ and $\rho_{12}(0)=\rho_{21}(0)^{*}=0$. Note that this is a very small perturbation of the thermal configuration. Parameters are chosen according to $\Delta \omega=7.25 \mathrm{GHz}, a_{11}=1 / 2, a_{22}=-1 / 2$, $a_{21}=1 / 2 \sqrt{2}, a_{12}=\sqrt{1 / 2-a_{21}^{2}}$, and coupling constant $\lambda=1 / 10$. The (rather high) temperature is fixed by our choice $N=100$, higher then the critical temperature $N \sim 89.5$ (see text). However we stress that the qualitative behavior of the system evolution is general and does not depend on this particular choice, as it only suffices that general inequalities are satisfied (see text). Graph (a) reports the time evolution of the diagonal elements: they immediately decay to thermal occupations. Graphs (b) and (c) report the real and imaginary part for the polarization $\rho_{12}(t)$ : even such a small perturbation $\left(10^{-5}\right)$ is able to trigger nonzero polarizations that, eventually, diverge, leading to highly unphysical setting within few ps. In graph (d) we plot the eigenvalues for $\rho(t)$, with blue gridlines defining the physical region, between 0 and 1 . Not only the system does not relax to the thermal condition (from which it has only been slightly moved), but highly nonphysical conditions are found within few ps. This clearly shows that the CM approximation is not suitable for steady state analysis and large time description. It is important to stress that these divergences cannot possibly come from numerical rounding errors, as the model has been solved analytically, and only at that point the numerical value of the parameters has been substituted.

up in the most simple setting of the one particle sector of a two level system, interacting locally with a bosonic heat bath.

\section{Alternative formulation of the Markov limit: derivation of a "Quantum Fermi's golden rule"}

Motivated by the intrinsic limitations of the CM approach, it is now our aim to propose an alternative formulation of the Markov limit, able to provide a Lindblad-like scattering superoperator (see Eq. (14)), thus preserving the positive-definite character of our density matrix, presented in a clear and physical formulation (see $[20,21,33]$ for abstract approaches). To this end, let us go back to the integro-differential equation (4). As previously recalled, the crucial step in the standard derivation is to replace $\rho^{i}\left(t^{\prime}\right)$ with $\rho^{i}(t)$. However, since in the adiabatic limit the time variation of the density matrix within the interaction picture is negligible, the latter can be evaluated not only at time $t$, but at any time between $t_{\circ}$ and $t$. Based on this crucial remark, what we propose is the following time symmetrization: given the two times $t^{\prime}$ and $t$, we shall introduce the "average" or "macroscopic" time $T=\frac{t+t^{\prime}}{2}$ and the "relative" time $\tau=t-t^{\prime}$. The basic idea is that the relevant time characterizing/describing our effective system evolution is the macroscopic time $T$. Following this spirit, it is easy to rewrite the second-order contribution in equation (4) in terms of the new time variables $T$ and $\tau$ :

$$
\begin{aligned}
\frac{d}{d T} \rho^{i}(T) & =-\int_{0}^{t-t_{\circ}} d \tau \\
\times & {\left[\mathcal{H}^{i}\left(T+\frac{1}{2} \tau\right),\left[\mathcal{H}^{i}\left(T-\frac{1}{2} \tau\right), \rho^{i}\left(T-\frac{1}{2} \tau\right)\right]\right] . }
\end{aligned}
$$

In the spirit of the adiabatic approximation previously recalled, the density-matrix operator $\rho^{i}$ can be taken out of the time integral and evaluated at the current time $T$. Moreover, it is convenient to replace the finite-domain time integration over $\tau$ by introducing a corresponding Gaussian correlation function $e^{-\frac{\tau^{2}}{2 \bar{t}^{2}}}$ whose width $\bar{t}$ may be regarded as a safe overestimation of the so-called "collision duration" [27]; indeed, for $t-t$ 。 greater than the collision duration the time integration may be safely extended up to infinity (see below). Focusing on the skew-adjoint part of equation (117), i.e., the so-called scattering part (the self-adjoint part is just an energy-renormalization term and does not threaten positivity), we get:

$$
\begin{aligned}
& \frac{d}{d T} \rho^{i}(T)=-\frac{1}{2} \int_{-\infty}^{\infty} d \tau \\
& \times e^{-\frac{\tau^{2}}{2 \bar{t}^{2}}}\left[\mathcal{H}^{i}\left(T+\frac{1}{2} \tau\right),\left[\mathcal{H}^{i}\left(T-\frac{1}{2} \tau\right), \rho^{i}(T)\right]\right] .
\end{aligned}
$$

We stress how the proposed time symmetrization gives rise to a fully symmetric superoperator, compared to the strongly asymmetric Markov superoperator [19] in equation (7).

The second crucial step in order to get a genuine Lindblad superoperator for the global dynamics is to exploit once again the slowly-varying character of the density-matrix operator $\rho^{i}$ on the right-hand side of equation (118). The key idea is to perform on both sides of equation (118) a so-called temporal "coarse graining", i.e., a weighted time average on a so-called microscopic scale, a scale over which the variation of $\rho^{i}(T)$ is negligible. Since in the small and intermediate coupling regime such timescale is fully compatible with the collision-duration timescale $\bar{t}$, we shall perform such time average employing once 
again a Gaussian correlation function of width $\frac{\bar{t}}{2}$, i.e.,

$$
\begin{aligned}
& \frac{d \rho^{i}}{d T}(T)=-\frac{1}{\sqrt{2 \pi \bar{t}}} \iint_{-\infty}^{\infty} d T^{\prime} d \tau e^{-\frac{4 T^{\prime 2}+\tau^{2}}{2 \bar{t}^{2}}} \\
& \quad \times\left[\mathcal{H}^{i}\left(T+T^{\prime}+\frac{\tau}{2}\right),\left[\mathcal{H}^{i}\left(T+T^{\prime}-\frac{\tau}{2}\right), \rho^{i}(T)\right]\right] .
\end{aligned}
$$

Moving back to the original Schrödinger picture and combining the two Gaussian distributions, the above equation can be rewritten in the following compact form:

$$
\frac{d \rho}{d T}=-\frac{1}{2}[\mathcal{L},[\mathcal{L}, \rho]]
$$

with

$$
\mathcal{L}=\left(\frac{2}{\pi \bar{t}^{2}}\right)^{\frac{1}{4}} \int_{-\infty}^{\infty} d t^{\prime} \mathcal{H}^{i}\left(t^{\prime}\right) e^{-\frac{t^{\prime 2}}{\bar{t}^{2}}} .
$$

This is the genuine Lindblad-like superoperator we were looking for; indeed, the operator $\mathcal{L}$ is always Hermitian, and such effective dynamics is positive-definite (see Eq. (14)).

Let us finally rewrite the new Markov superoperator (120) in our noninteracting basis $\lambda$, defined by the (possibly generalized) eigenvectors of $H_{\circ}$ : we obtain an effective equation of motion of the form

$\frac{d \rho_{\lambda_{1} \lambda_{2}}}{d t}=\frac{1}{2} \sum_{\lambda_{1}^{\prime} \lambda_{2}^{\prime}}\left[\mathcal{P}_{\lambda_{1} \lambda_{2}, \lambda_{1}^{\prime} \lambda_{2}^{\prime}} \rho_{\lambda_{1}^{\prime} \lambda_{2}^{\prime}}-\mathcal{P}_{\lambda_{1}^{\prime} \lambda_{1}^{\prime}, \lambda_{1} \lambda_{2}^{\prime}}^{*} \rho_{\lambda_{2}^{\prime} \lambda_{2}}\right]+$ H.c.

with symmetrized quantum scattering rates

$$
\begin{aligned}
& \mathcal{P}_{\lambda_{1} \lambda_{2}, \lambda_{1}^{\prime} \lambda_{2}^{\prime}}=2 \pi \hbar H_{\lambda_{1} \lambda_{1}^{\prime}}^{\prime} H_{\lambda_{2} \lambda_{2}^{\prime}}^{\prime *} \\
& \quad \times \frac{1}{\sqrt{2 \pi} \bar{\epsilon}} \exp \left\{-\frac{\left(\epsilon_{\lambda_{1}}-\epsilon_{\lambda_{1}^{\prime}}\right)^{2}+\left(\epsilon_{\lambda_{2}}-\epsilon_{\lambda_{2}^{\prime}}\right)^{2}}{4 \bar{\epsilon}^{2}}\right\} .
\end{aligned}
$$

Here, $\hbar$ has been shown explicitly (but remember that $H_{\lambda_{1} \lambda_{1}^{\prime}}^{\prime}$ is still measured in $\left.H z\right), \epsilon_{\lambda}=\hbar \omega_{\lambda}$ denotes the energy corresponding to the noninteracting state $\lambda$, and $\bar{\epsilon}=\hbar \bar{\omega}=\frac{\hbar}{\bar{t}}$ is a measure of the energy uncertainty in the interaction process induced by our temporal coarse graining.

The well-known semiclassical or Boltzmann theory [1] can be easily derived from the quantum formulation presented so far, by introducing the so-called diagonal or semiclassical approximation. The latter corresponds to neglecting all non-diagonal density-matrix elements (and therefore any quantum-mechanical phase coherence between the generic states $\lambda_{1}$ and $\lambda_{2}$ ), i.e.,

$$
\rho_{\lambda_{1} \lambda_{2}}=f_{\lambda_{1}} \delta_{\lambda_{1} \lambda_{2}}
$$

where the diagonal elements $f_{\lambda}$ describe the semiclassical distribution function over our noninteracting basis states.

Within such approximation scheme, the effective quantum equation (122) reduces to the well-known Boltzmann equation:

$$
\frac{d f_{\lambda}}{d t}=\sum_{\lambda^{\prime}}\left(P_{\lambda \lambda^{\prime}} f_{\lambda^{\prime}}-P_{\lambda^{\prime} \lambda} f_{\lambda}\right)
$$

and in the completed-collision limit $(\bar{\epsilon} \rightarrow 0)$ the scattering superoperator in equation (123) reduces to the standard scattering rates given by the well-known Fermi's Golden Rule [16]:

$$
P_{\lambda \lambda^{\prime}}=\mathcal{P}_{\lambda \lambda, \lambda^{\prime} \lambda^{\prime}}=\frac{2 \pi}{\hbar}\left|H_{\lambda \lambda^{\prime}}^{\prime}\right|^{2} \delta\left(\epsilon_{\lambda}-\epsilon_{\lambda^{\prime}}\right) .
$$

So, we have shown here that our generalized scattering operators include the Fermi's Golden Rule as the diagonal case, just as the CM superoperator did, as we have shown before.

\section{Partial trace projection of the new Markov superoperator}

We defer the reader back to Section 3 for notation and formalism used here, where we are going to write our Lindblad, but still global, superoperator, into its projected form, for the partial trace projection, and prove that the result still has a Lindblad form.

More specifically, let us start by applying the trace over the bath coordinates to equation (120); by expanding the double commutator and employing the factorization scheme in equation (24) we get:

$$
\begin{aligned}
\frac{d \rho^{e}}{d T} & =-\frac{1}{2} \operatorname{tr}\left\{\left[\mathcal{L},\left[\mathcal{L}, \rho^{e} \rho^{b}\right]\right]\right\}_{b} \\
& =-\frac{1}{2} \operatorname{tr}\left\{\mathcal{L} \mathcal{L} \rho^{e} \rho^{b}\right\}_{b}-\frac{1}{2} \operatorname{tr}\left\{\rho^{e} \rho^{b} \mathcal{L} \mathcal{L}\right\}_{b}+\operatorname{tr}\left\{\mathcal{L} \rho^{e} \rho^{b} \mathcal{L}\right\}_{b}
\end{aligned}
$$

To evaluate the results of the trace, it is imperative to use the explicit form of the interaction Hamiltonian $H^{i}(t)$ in equation (31). We obtain:

$$
\begin{aligned}
\frac{d \rho^{e}}{d T}= & -\frac{1}{2} \sum_{ \pm} \int d \mathbf{q}\left(N_{\mathbf{q}}+\frac{1}{2} \pm \frac{1}{2}\right) \mathcal{L}_{\mathbf{q}}^{ \pm \dagger} \mathcal{L}_{\mathbf{q}}^{ \pm} \rho^{e} \\
& -\frac{1}{2} \sum_{ \pm} \int d \mathbf{q}\left(N_{\mathbf{q}}+\frac{1}{2} \pm \frac{1}{2}\right) \rho^{e} \mathcal{L}_{\mathbf{q}}^{ \pm \dagger} \mathcal{L}_{\mathbf{q}}^{ \pm} \\
& +\sum_{ \pm} \int d \mathbf{q}\left(N_{\mathbf{q}}+\frac{1}{2} \pm \frac{1}{2}\right) \mathcal{L}_{\mathbf{q}}^{ \pm} \rho^{e} \mathcal{L}_{\mathbf{q}}^{ \pm \dagger}
\end{aligned}
$$

with

$$
\mathcal{L}_{\mathbf{q}}^{ \pm}=\left(\frac{2}{\pi \bar{t}^{2}}\right)^{\frac{1}{4}} \int_{-\infty}^{\infty} d t H_{\mathbf{q}}^{ \pm}(t) e^{-\frac{t^{2}}{\bar{t}^{2}}}
$$

A closer inspection of equation (128) shows that this effective scattering superoperator is again Lindblad-like. Indeed, by rewriting the first two terms via a corresponding anticommutator, equation (128) will exhibit exactly the form of the Lindblad superoperator in equation (13), namely:

$$
\begin{aligned}
\frac{d \rho^{e}}{d T}= & \sum_{ \pm} \int d \mathbf{q}\left(N_{\mathbf{q}}+\frac{1}{2} \pm \frac{1}{2}\right) \\
& \times\left(-\frac{1}{2}\left\{\mathcal{L}_{\mathbf{q}}^{ \pm \dagger} \mathcal{L}_{\mathbf{q}}^{ \pm}, \rho^{e}\right\}+\mathcal{L}_{\mathbf{q}}^{ \pm} \rho^{e} \mathcal{L}_{\mathbf{q}}^{ \pm \dagger}\right)
\end{aligned}
$$


This result is extremely important: as for the case of the global (system plus reservoir) treatment presented in Section 5, also for the reduced or electronic description our effective dynamics is Lindblad-like, thus preserving the positive-definite character of the electronic density-matrix operator $\rho^{e}$. However, while for the global case we deal with a Hermitian Lindblad operator $\mathcal{L}$ (corresponding to the double-commutator generator in equation (14), within our reduced description the effective Lindblad operators $\mathcal{L}_{q}^{ \pm}$(corresponding to the generalized Lindblad generator in Eq. (13)) are non-Hermitian quantities.

Let us finally rewrite the scattering superoperator in equation (128) in our electronic basis $\{|\alpha\rangle\}$. By denoting with

$$
H_{\alpha_{1} \alpha_{2}}^{\mathbf{q}}=\left\langle\alpha_{1}\left|H_{\mathbf{q}}\right| \alpha_{2}\right\rangle
$$

the matrix elements of the electronic interaction operator $H_{\mathbf{q}}$ in equation (19), we get:

$$
\frac{d \rho_{\alpha_{1} \alpha_{2}}^{e}}{d T}=\frac{1}{2} \sum_{\alpha_{1}^{\prime} \alpha_{2}^{\prime}}\left[\mathcal{P}_{\alpha_{1} \alpha_{2}, \alpha_{1}^{\prime} \alpha_{2}^{\prime}}^{e} \rho_{\alpha_{1}^{\prime} \alpha_{2}^{\prime}}^{e}-\mathcal{P}_{\alpha_{1}^{\prime} \alpha_{1}^{\prime}, \alpha_{1} \alpha_{2}^{\prime}}^{e *} \rho_{\alpha_{2}^{\prime} \alpha_{2}}^{e}\right]+\text { H.c. }
$$

with generalized electronic scattering rates

$$
\begin{aligned}
& \mathcal{P}_{\alpha_{1} \alpha_{2}, \alpha_{1}^{\prime} \alpha_{2}^{\prime}}^{e}=2 \pi \sum_{ \pm} \int d \mathbf{q}\left(N_{\mathbf{q}}+\frac{1}{2} \pm \frac{1}{2}\right) H_{\alpha_{1} \alpha_{1}^{\prime}}^{\mathbf{q}} H_{\alpha_{2} \alpha_{2}^{\prime}}^{\mathbf{q} *} \\
& \times \frac{1}{\sqrt{2 \pi} \bar{\omega}} e^{-\frac{\left(\omega_{\alpha_{1}}-\omega_{\alpha_{1}^{\prime}} \pm \omega_{b}(\mathbf{q})\right)^{2}+\left(\omega_{\alpha_{2}}-\omega_{\alpha_{2}^{\prime}} \pm \omega_{b}(\mathbf{q})\right)^{2}}{4 \bar{\omega}^{2}}} .
\end{aligned}
$$

Again, the above scattering superoperator can be regarded as the quantum-mechanical generalization of the conventional Fermi's golden rule describing our electronic quantum subsystem interacting with a quasiparticle environment. Indeed, in the semiclassical limit $\left(\rho_{\alpha \alpha^{\prime}}^{e}=f_{\alpha}^{e} \delta_{\alpha \alpha^{\prime}}\right)$ and in the completed-collision limit $(\bar{\omega} \rightarrow 0)$, the effective quantum equation (132) reduces to the following Boltzmann equation for the electronic subsystem:

$$
\frac{d f_{\alpha}}{d T}=\sum_{\alpha^{\prime}}\left(P_{\alpha \alpha^{\prime}}^{e} f_{\alpha^{\prime}}^{e}-P_{\alpha^{\prime} \alpha}^{e} f_{\alpha}^{e}\right)
$$

with

$$
\begin{aligned}
P_{\alpha \alpha^{\prime}}^{e}= & \mathcal{P}_{\alpha \alpha, \alpha^{\prime} \alpha^{\prime}}^{e} \\
= & 2 \pi \sum_{ \pm} \int d \mathbf{q}\left(N_{\mathbf{q}}+\frac{1}{2} \pm \frac{1}{2}\right)\left|H_{\alpha \alpha^{\prime}}^{\mathbf{q}}\right|^{2} \\
& \times \delta\left(\omega_{\alpha}-\omega_{\alpha^{\prime}} \pm \omega_{b}(\mathbf{q})\right) .
\end{aligned}
$$

We finally stress that, as for the general result in equation (123), our Lindblad-like scattering superoperator corresponds again to fully symmetric scattering rates; this is a distinguished advantage of the proposed adiabaticdecoupling procedure, compared to the conventional (i.e., non-Lindblad) quantum scattering rates discussed in reference [32].

\section{Generalization to other reduction/projection schemes}

As pointed out previously, the proposed theoretical formulation becomes meaningful and applicable only when a well-defined subsystem of interest is identified (together with a corresponding infinite-dimensional environment), so that its completed collision time $\bar{t}$ can be estimated, and our (irreversible) semigroup dynamics can correctly describe the projected (but fully reversible) exact Hamiltonian dynamics. Indeed, this is exactly the case of the effective electronic description previously considered (corresponding to a so-called partial-trace projection).

As final crucial step, we shall show that our conclusions about positivity remain valid no matter how the subsystem is chosen, i.e. not only for the particular case of the electronic description in Section 3. To this end, we notice that the usual partial-trace projection, when viewed in Heisenberg picture, is of the form $P_{0}: \sum_{n} A_{n} \otimes B_{n} \mapsto$ $\left(\sum_{n} \operatorname{Tr}\left(\omega B_{n}\right) A_{n}\right) \otimes 1$, where $\omega$ is the "environment" density matrix, $A_{n}$ and $B_{n}$ are, respectively, generic system and environment observables. From this structure, it follows that the partial trace is completely positive (see, e.g., Ref. [12]). Moreover, the projected observables, all being of the form $A \otimes 1$, constitute a subalgebra of the globalobservable algebra. Based upon these two key remarks, let us now consider a generic projection $P_{0}$ on a subalgebra $\mathcal{X}$ of the space of observables in Heisenberg picture, which is also a completely positive map, and let $\left\{V_{\alpha}\right\}$ be its Kraus decomposition [34], so that $P_{0} A=\sum_{\alpha} V_{\alpha}^{\dagger} A V_{\alpha}$. Then one could easily verify that $\mathcal{X}$ is made by observables that commute with each of the $V_{\alpha}$ and $V_{\alpha}^{\dagger}$. We now observe that, due to its symmetry, our generator keeps the same form of equation (120) also in Heisenberg picture: by projecting the latter with $P_{0}$, and using the completeness relation $\sum_{\alpha} V_{\alpha}^{\dagger} V_{\alpha}=1$, one can easily write the form for the subsystem's generator in Schrödinger picture, dual to the projected dynamics on the subalgebra $\mathcal{X}$ :

$$
\frac{d}{d T} \widehat{\rho}=-\frac{1}{2} \sum_{\alpha \beta}\left\{\widehat{D}_{\alpha \beta}^{\dagger} \widehat{D}_{\alpha \beta}, \widehat{\rho}\right\}+\sum_{\alpha \beta} \widehat{D}_{\alpha \beta} \widehat{\rho} \widehat{D}_{\alpha \beta}^{\dagger} .
$$

Here the ( $\bar{t}$-dependent) "quantum transition amplitude" operators are given, according to equation (121), by

$$
\widehat{D}_{\alpha \beta}=\widehat{V}_{\alpha} \widehat{\mathcal{L}} \widehat{V}_{\beta}
$$

This form is again Lindblad-like (see Eq. (13)), thus showing indeed that we have obtained the generator of the completely positive Quantum Dynamical Semigroup we were looking for. Moreover, the effort we made to consider this rather abstract class of projection is completely justified, as, for example, it paves the way for a new quantumtransport formalism: suppose that $P$ is a projection in our Hilbert space that identifies, say, a one-dimensional nano-device, and let $Q_{l}$ and $Q_{r}$ project on the left and right contact respectively (obviously $P+Q_{l}+Q_{r}=1$ ). Then $P_{0} A=P A P+Q_{l} A Q_{l}+Q_{r} A Q_{r}$ does belong to the class of projections we have just studied, but the chosen subsystem does not come from a partial trace, nor does 
it have finite dimensions or discrete spectral properties: its weak-coupling dynamics needs the full power of our theory, in contrast with the previous ones $[15,19]$.

As final remark, it is important to point out that the electronic description presented in Section 3 refers, in general, to a many-electron subsystem, i.e., the generic electronic state $|\alpha\rangle$ describes the exact eigenstate of $N$ interacting electron system. However, for most of the key properties of semiconductor quantum devices, such a many-electron treatment is not required, and the latter is replaced by a so-called single-electron description. This corresponds to replace the many-electron density-matrix operator $\rho^{e}$ with a single-electron density matrix.

As for the case of the partial trace over the quasiparticle coordinates, such many-body versus single-electron reduction operation does not commute with the scattering superoperator in equation (132). As discussed extensively in reference [5], to get a closed equation of motion for the single-electron density matrix, an additional approximation is needed, namely the so-called mean-field approximation. However, the resulting effective equation is non-linear and for this reason the investigation of the positivity of the single-electron density matrix is still an open problem, far beyond the scope of the present paper.

\section{Summary and conclusions}

In summary, we have presented a general density-matrix description of energy-dissipation and decoherence phenomena in open quantum devices. More specifically, contrary to the conventional single-particle correlation expansion, we have investigated the effect of the Markov limit, before considering/performing any reduction procedure. Our fully operatorial approach has allowed us to better identify the general properties of the scattering superoperators entering our effective quantum-transport theory at various description levels.

In particular, we have shown very important intrinsic limitations of the CM approach when employed for large time or steady state analysis. Even neglecting the renormalisation part gives a good steady state (thermal), which however is highly unstable: arbitrarily small perturbations need not relax thermally back to the steady solution, and may well lead to divergent polarizations. Our results show that these intrinsic limitations already show up in the most simple setting of the one particle sector of a two level system, interacting locally with a bosonic heat bath.

To overcome this crucial limitation, we have identified an alternative and more general adiabatic procedure which, on the one hand, in the semiclassical limit reduces to the standard Fermi's golden rule, and, on the other hand, describes a genuine Lindblad evolution, thus providing a reliable/robust treatment of energy-dissipation and dephasing in state-of-the-art semiconductor quantum devices. We stress that our formulation generalizes preexisting theories significantly, as it gives a positive dynamics for a considerably large class of projections, i.e. ways to chose the subsystem, irrespective of the subsystem's dimension or spectral properties. In turn, on one side this allows to investigate subsystems with both discrete and continuous spectra, a feature largely shared by mesoscale electronic and opto-electronic quantum devices; on the other side, it suggests a new way to treat electrical contacts for quantum devices, thus opening up the exciting possibility of a new formalism for Quantum Transport.

At this point an important remark is in order. As discussed extensively in reference [32], also for the simplest case of a standard two-level system - i.e., a generic quantum bit - the standard Markov superoperator predicts a non-trivial coupling between level population and polarization described by the so-called $T_{3}$ contributions. In contrast, for a two-level system coupled to its environment, the proposed quantum Fermi's golden rule does not predict any $T_{3}$ coupling term (they vanish in the completed collision limit $\bar{\epsilon} \rightarrow 0$ ), thus providing a rigorous derivation of the well-known and successfully employed $T_{1} T_{2}$ dephasing model [3]. In general in fact, one could show that for subsystems with discrete spectra, the completedcollision limit reduces to Davies' theory [15]. However, for subsystems with continuous spectra, such limit is not defined, but for all finite collision times $\bar{t}>0$ the proposed approach gives $\bar{t} \sim T_{3}$, so $T_{3}$ contributions are indeed present, but they become less and less important as the collision time $\bar{t}$ is raised, as it must be in the weak-coupling limit $^{2}$.

Finally, it is imperative to stress that in the presence of a strong system-environment interaction the adiabatic decoupling investigated so far needs to be replaced by more realistic treatments, expressed via non-Markovian integrodifferential equations of motion [5] (i.e., with "memory effects"). Again, while for purely atomic and/or photonic systems it is possible to identify effective non-Markovian evolution operators [35], for solid-state quantum devices this is still an open problem.

\section{References}

1. See, e.g., C. Jacoboni, P. Lugli, The Monte Carlo Method for Semiconductor Device Simulations (Springer, Wien, 1989)

2. See, e.g., Hot Carriers in Semiconductor Nanostructures: Physics and Applications, edited by J. Shah (Academic Press inc., Boston, 1992); F.A. Buot, Phys. Rep. 234, 73 (1993); F.A. Buot, Theory of Transport Properties of Semiconductor Nanostructures, edited by E. Schöll (Chapman and Hall, London, 1998)

3. See, e.g., J. Shah, Ultrafast Spectroscopy of Semiconductors and Semiconductor Nanostructures (Springer, Berlin, 1996)

4. See, e.g., D. Bimberg, M. Grundmann, N.N. Ledentsov, Quantum Dot Heterostructures (Wiley, Chichester, 1998);

${ }^{2}$ To compare with the exact dynamics in the weak-coupling limit, the collision time has to finally be brought back to infinity: this in turn may be accomplished by a scaling property of the form $\bar{t}(g) \sim g^{-\xi} \bar{T}$, for $g \sim 0$, where $g$ is the coupling constant, $\bar{T}$ is a fixed reference collision time, and $\xi>0$. 
L. Jacak, P. Hawrylak, A. Wojs, Quantum Dots (Springer, Berlin, 1998); S.M. Reimann, M. Manninen, Rev. Mod. Phys. 74, 1283 (2002)

5. See, e.g., F. Rossi, T. Kuhn, Rev. Mod. Phys. 74, 895 (2002)

6. See, e.g., W. Frensley, Rev. Mod. Phys. 62, 3 (1990)

7. See, e.g., Physics of Quantum Electron Devices, edited by F. Capasso (Springer, Berlin, 1990)

8. See, e.g., C. Gmachl, F. Capasso, D.L. Sivco, A.Y. Cho, Rep. Prog. Phys. 64, 1533 (2001); R. Köhler, A. Tredicucci, F. Beltram, H.E. Beere, E.H. Linfield, A. Giles Davies, D.A. Ritchie, R.C. Iotti, F. Rossi, Nature 417, 156 (2002); R.C. Iotti, F. Rossi, Rep. Prog. Phys. 68, 2533 (2005)

9. See, e.g., Semiconductor Macroatoms: Basic Physics and Quantum-device Applications, edited by F. Rossi (Imperial College Press, London, 2005); Focus on Solid State Quantum Information, edited by R. Fazio, New J. Phys. 7 (2005)

10. See e.g., M.O. Scully, M.S. Zubairy, Quantum Optics (Cambridge University Press, Cambridge, 1997)

11. See, e.g., E.B. Davies, Quantum Theory of Open Systems (Academic Press, London, 1976)

12. G. Lindblad, Commun. Math. Phys. 48, 119 (1976)

13. See, e.g., S.G. Schirmer, A.I. Solomon, Phys. Rev. A 70, $022107(2004)$

14. R. Dümcke, H. Spohn, Z. Phys. B 34, 419 (1979)

15. E.B. Davies, Commun. Math. Phys. 39, 91 (1974)

16. See, e.g., E. Fermi, Nuclear Physics (University of Chicago Press, 1950)

17. R. Alicky, International Journal of Theoretical Physics $\mathbf{1 6}$ 351 (1977)

18. See, e.g., H. Spohn, Rev. Mod. Phys. 53, 569 (1980)

19. E.B. Davies, Math. Ann. 219, 147 (1976)

20. D. Taj, F. Rossi, Phys. Rev. A 78, 052113 (2008)
21. D. Taj, Weak Coupling Limit I: a Contraction Semigroup for Infinite Subsystems, e-print: [arXiv:0905.1012v1] (2009)

22. See e.g. A. Vagov, V.M. Axt, T. Kuhn, Phys. Rev. B 67, 115338 (2003)

23. See, e.g., L.P. Kadanoff, G. Baym, Quantum Statistical Mechanics (Benjamin, New York, 1962)

24. See, e.g., L.V. Keldysh, Sov. Phys. JETP 20, 1018 (1965)

25. See, e.g., H. Haug, A.-P. Jauho, Quantum Kinetics in Transport and Optics of Semiconductors (Springer, Berlin, 1996)

26. See, e.g., R. Lake, S. Datta, Phys. Rev. B 45, 6670 (1992); C. Rivas et al., Appl. Phys. Lett. 78, 814 (2002)

27. See, e.g., F. Rossi, R. Brunetti, C. Jacoboni, in Hot Carriers in Semiconductor Nanostructures: Physics and Applications, edited by J. Shah (Academic Press Inc., Boston, 1992), p. 153

28. See, e.g., N.N. Bogoliubov, Lectures on Quantum Statistics (Gordon and Breach, New York, 1967)

29. See, e.g., V.M. Axt, S. Mukamel, Rev. Mod. Phys. 70, 145 (1998)

30. See, e.g., R. Brunetti, C. Jacoboni, F. Rossi, Phys. Rev. B 39, 10781 (1989); P. Bordone, M. Pascoli, R. Brunetti, A. Bertoni, C. Jacoboni, Phys. Rev. B 59, 3060 (1999)

31. See, e.g., T. Kuhn, in Theory of Transport Properties of Semiconductor Nanostructures, edited by E. Schöll (Chapman and Hall, London, 1998), p. 173

32. R.C. Iotti, E. Ciancio, F. Rossi, Phys. Rev. B 72, 125347 (2005)

33. D. Taj, Weak Coupling Limit II: on the Quantum FokkerPlanck Equation, e-print: [arXiv:0905.1019v1] (2009)

34. K. Kraus, Ann. Phys. 64, 311 (1970)

35. See, e.g., A.A. Budini, Phys. Rev. A 74, 053815 (2006)

36. T. Grange, R. Ferreira, G. Bastard, PRB 76, 241304(R) (2007) 